\title{
Reaction wheels desaturation using magnetorquers and static input allocation
}

\author{
Jean-François Trégouët, Denis Arzelier, Dimitri Peaucelle, Christelle Pittet and Luca Zaccarian
}

\begin{abstract}
Considering the most widely spread configuration of actuators for low orbit satellites, namely a set of reaction wheels and a set of magnetorquers, we revisit the classical "cross product control law" solution for achieving attitude stabilization and momentum dumping. We show how the classical solution has a quasi-cascade structure that, under a suitable input-tostate (ISS) assumption, can be stabilized by high gain, thereby making the actuators more inclined to saturate. Motivated by this, we propose a revisited version of this control law that transforms the quasi-cascade into a real cascade. Then we show that both strategies are such that the attitude control is affected by the momentum dumping, and that they both require a suitable ISS property. To overcome these drawbacks, we propose a new allocation-based controller which makes the attitude dynamics completely independent of the momentum dumping and induces global asymptotic stability without any ISS requirement. Several formal statements and simulation results support our discussions and highlight the pros and cons of the different control strategies.
\end{abstract}

Index Terms - aerospace control, attitude control, magnetorquers, reaction wheels desaturation, input allocation, Lyapunov methods.

\section{INTRODUCTION}

$\mathbf{T}$ $\mathrm{HE}$ attitude control problem has been a challenging issue for the scientific community for decades (see [1], [5], [24], [38] and references therein) and still constitutes an active area of research. Recently, the literature has been enriched by several remarkable solutions which achieve asymptotic stability in a global way [30], [26]. If these results are an important step, they rely on an abstract context for which the availability of an external actuation torque is assumed. However, when it comes to physical realization of this control torque by means of real actuators, solutions mainly rely on engineering consideration and can be largely improved.

Most of three-axis stabilized spacecrafts are equipped with angular momentum storage devices such as reaction wheels when high pointing accuracy is required [7]. Despite their known advantages [39], these momentum exchange systems may suffer from saturation limitations as well as static friction when approaching zero angular velocity. Thus, reaction wheels need a secondary attitude control system for momentum unloading and desaturation of the reaction wheels system (RWS). For small satellites in Low Earth orbits (LEO) magnetorquers

J.-F. Trégouët is with Université de Lyon, Laboratoire Ampère CNRS UMR 5005, INSA-Lyon; F-69621, Villeurbanne, France e-mail: jeanfrancois.tregouet@insa-lyon.fr. D. Arzelier, D. Peaucelle and L. Zaccarian are with CNRS, LAAS, 7 avenue du colonel Roche, F-31400 Toulouse, France and Univ de Toulouse, LAAS, F-31400 Toulouse, France. C. Pittet is with CNES, 18 avenue Edouard Belin, F-31401 Toulouse. L. Zaccarian is also with the Dipartimento di Ingegneria Industriale, University of Trento, Italy. provide a cheap, reliable and effective external torque for momentum desaturation purposes [3], [4], [8], [27], [33]. Earth observation satellites such as Spot4, Jason1 and 2, Demeter or as Corot for inertial pointing are thus equipped by magnetic and mechanical actuation systems [7], [28]. While reaction wheels are easily exploited because they can generate a torque in any desired direction at any time, magnetorquers rely on a more subtle principle: by generating a magnetic momentum that interacts with the geomagnetic field, a torque is created. However, as this field is not constant, due to the rotation of the satellite around the earth, the synthesis models are naturally almost periodically time-varying. In addition to that, at any given instant of time, the produced torque lies in the orthogonal plane to the instantaneous geomagnetic field leading to the non-controllability of the direction parallel to the local geomagnetic field vector. For these reasons, the control problem associated with the magnetorquers is recognized as a challenging one [27], [24].

Despite these limitations, engineers frequently resort to this kind of actuators, for cost-effectiveness, efficiency, reliability and weight reasons. Moreover, the combined action of the magnetorquers with the reaction wheels allows to regard the latter actuators as the main actuators for fine attitude control and the former ones as auxiliary actuators used for "desaturation" or "momentum dumping". A classical engineeringbased solution following this paradigm is the so-called "cross product control law" mentioned in [3], [4], [8], [33], where the problem is addressed within a linear approximation context.

In this paper we address the problem of designing a suitable centralized law for the reaction wheels and magnetorquers on an inertially pointing satellite orbiting at low altitude. More precisely, given an attitude stabilizer that has been initially designed disregarding the momentum dumping problem, we propose a control scheme managing the two actuators in such a way that momentum dumping is performed continuously throughout the maneuver while remaining completely hidden for the attitude dynamics that exactly follows the response induced by the prescribed attitude stabilizer. The paper's contributions are summarized next.

1) Our first contribution is to provide a rigorous interpretation (inspired by the formalization of [24], where only magnetorquers are considered) of the architecture behind the "cross product control law". The treatment helps formalizing this approach by highlighting its hidden assumptions. 2) As a second contribution, we propose a revisited version of the "cross product control law", which is shown to be valid in a wider range of practical situations. In particular, we show a peculiar feedback structure arising from this controller, 
which can be converted (using the proposed revisited law) into a more convenient cascaded form consisting of an upper subsystem whose state is the total angular momentum and a lower subsystem whose state is the satellite attitude (position and speed). We discuss and show by simulations that the above cascaded structure is not desirable due to the fact that the regulation of the angular momentum of the wheels perturbs the attitude control system. 3) Our third and main contribution is then given and consists in a novel control scheme that is capable of reversing the cascaded scheme by way of static input allocation. Input allocation techniques address the problem of suitably assigning the low level actuators input, based on a higher level control effort requested by the control system [18]. Their use is especially suited in the presence of redundant plant inputs from the point of view of the main control task. Then the allocation can be performed in such a way to optimize a cost function related to a lower priority secondary task (see [32] for an interesting collection of results in the aerospace, marine and terrestrial vehicles). Within the attitude control setting described above, it appears natural to regard the primary goal as the attitude stabilization goal and the secondary goal as the reaction wheels desaturation goal. To this end, the employed allocation scheme may be selected as a static one (this is the structure of most of the existing techniques, well surveyed in [18]) or a dynamic one (following, e.g., the paradigm in [40] or the more recent developments in [31], [11]). Note that the use of an allocation strategy for the engineering problem described here has been also proposed in [10]. However, the suggested distribution of the total control effort does not address the dynamics of the angular momentum of the wheels, which is instead the goal of the allocation scheme proposed in this paper.

In a nutshell, the allocation-based control scheme proposed here completely decouples the attitude stabilization task from the angular momentum of the wheels and is therefore capable of stabilizing the attitude dynamics following a prescribed law that can be designed disregarding the momentum dumping task. This goal is achieved by the cascaded structure of the new scheme where the upper subsystem, consisting in the (undisturbed) attitude stabilization loop, drives the lower subsystem, which performs the (lower priority) task of desaturating the reaction wheels. A preliminary version of this paper was presented in [37]. Here, as compared to [37], we treat a more general scenario with non-periodic local geomagnetic fields, we provide several statements and proofs of the stability properties of the proposed schemes, and we provide revised and improved simulation tests based on realistic data from satellite missions.

The paper is structured as follows. In Section II we introduce the satellite model, some preliminary facts about global attitude stabilization and formalize our problem statement. In Section III we explain and interpret the "cross product control law", provide a revisited version of it and establish its formal properties. In Section IV we propose the new control strategy based on static allocation. In Section V we provide comparative simulation results for the two controllers introduced in Sections III and IV. Finally, in Section VI we give some concluding remarks.
Notation: Given any vectors $v, w \in \mathbb{R}^{3}$, the matrix $v^{\times} \in$ $\mathbb{R}^{3 \times 3}$ is a skew-symmetric matrix defined in such a way that the vector product between $v$ and $w$ satisfies $v \times w=v^{\times} w$, i.e. $v^{\times}=\left[\begin{array}{ccc}0 & -v_{z} & v_{y} \\ v_{z} & 0 & -v_{x} \\ -v_{y} & v_{x} & 0\end{array}\right]$ for $v=\left[\begin{array}{lll}v_{x} & v_{y} & v_{z}\end{array}\right]^{T}$. The identity quaternion is denoted by $q_{\circ}=\left[\begin{array}{lll}0 & 0 & 0\end{array}\right]^{T}$. The set $\mathbb{R}_{\geq 0}$ denotes the non-negative reals while $\mathbb{Z}_{\geq 0}$ denotes the non-negative integers. The identity matrix of size $n \times n$ is written $\mathbf{1}_{n}$. Depending on its argument, the bars $|\cdot|$ refer to the absolute value of a scalar, the Euclidean norm of a vector or the induced $l_{2} /$ spectral-norm of a matrix. The superscript ${ }^{[I]}$ indicates that the related vector is expressed in the inertial frame. Otherwise, the body-fixed frame is considered.

\section{Preliminaries And PRoblem Statement}

Figure 1 represents the scenario addressed in this paper, namely an inertially pointing satellite equipped with two actuator sets:

1) the reaction wheels that are capable of exerting a triple of torques spanning all the degrees of freedom of the attitude dynamics but suffer from the drawback of possibly experiencing a gradual increase of their spinning speed, due to their inability to alter the total momentum of the satellite affected by external disturbances;

2) the magnetorquers that are capable of exerting a rank deficient torque on two out of the three degrees of freedom of the attitude dynamics due to the fact that they interact with the geomagnetic field; this second actuator may affect the total momentum of the satellite and therefore can be used to suitably stabilize the rotational speed of the wheels, even though the rank deficient nature of the generated torque is a challenge to be addressed.

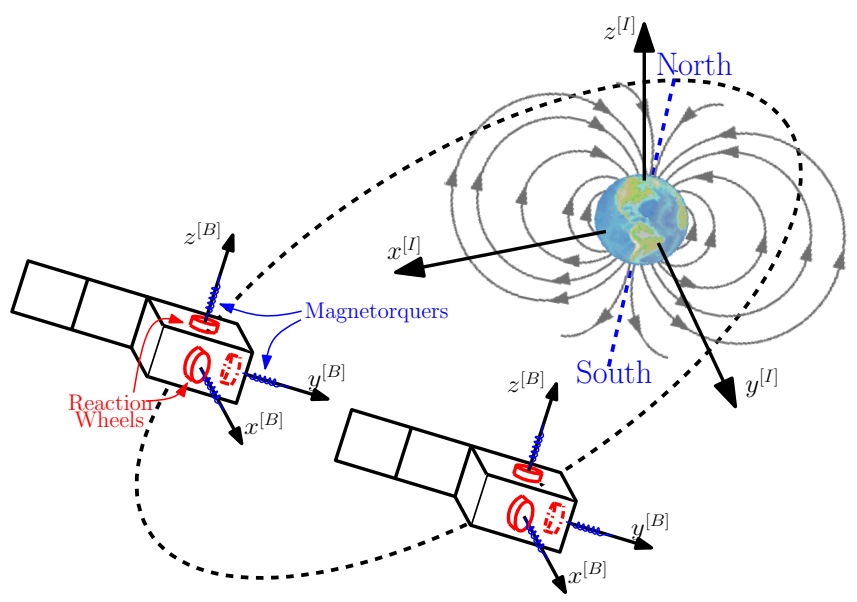

Fig. 1. An inertially pointing satellite orbiting around the earth and equipped with reaction wheels and magnetorquers.

Within the above context, we address in this paper the goal of suitably using the redundancy available in the two sets of actuators to stabilize the attitude of the satellite while simultaneously regulating the rotational speed of the wheels (momentum dumping). Note that attitude stabilization and momentum dumping are potentially conflicting goals because 
attitude control requires to speed up (or decelerate) the wheels, which may badly interact with the momentum dumping performed by the magnetorquers. From an engineering standpoint, the problem addressed and solved in this paper can be formulated as follows.

Problem 1. Given a spacecraft equipped with reaction wheels and magnetorquers actuators, as represented in Figure 1, design a control strategy for driving the two sets of actuators to ensure simultaneously that the body frame is aligned with the inertial frame (attitude stabilization) and that the angular momenta of the reaction wheels are stabilized at a given reference value.

As customary in the case of redundancy of actuators, the engineering problem described in Problem 1 is usually addressed in two steps. First, a suitable attitude stabilizer with respect to given attitude specifications is obtained. It is based on an abstract model that does not take the reaction wheels saturation issue into account. Then, an additional control layer, implementing this control law for the retained actuation equipment, is designed. We stress that this paper is mainly concerned with the second step. Thus, all our derivations are valid for any stabilizer as long as it satisfies some mild regularity properties. Nevertheless, for illustration purposes, and to show the potential of our scheme to provide global results on nonlinear models, we use the attitude controller proposed in [26].

To suitably address and solve Problem 1, we give in this section the essential material to formulate it in mathematical terms. First, the dynamics of the satellite model are described in Section II-A. Then in Section II-B we recall the global attitude stabilizer proposed in [26] and prove some additional properties that were not given in [26]. Then in Section II-C we introduce some assumptions on the satellite orbit that enable us, together with the other derivations of the section, to mathematically formalize the engineering Problem 1.

Remark 1. We emphasize that the design problem stated in Problem 1 addresses the issue of saturation of the angular speed of the wheels but should take into account the maximum torque that can be exerted both by the magnetorquers and by the reaction wheels actuators. While we do take into account all these different types of saturations in the simulations carried out in Section V, we do not take torque saturations into account in our mathematical analysis. Indeed, taking saturation into account at a design level is a challenging nonlinear control problem that would most likely lead to conservative bounds and possibly sacrifice the small signal behavior. A viable approach that does not sacrifice local performance is that of anti-windup design [41], [35], which poses nontrivial theoretical difficulties when applied to nonlinear saturated plants (see, e.g., the discussion in the recent paper [21]). Since our allocation-based solution allows for any attitude stabilizer, one may actually take saturation into account in the attitude stabilization design and then apply our allocationbased desaturation scheme. Clearly, the challenging problem of designing such a stabilizer is beyond the scope of this paper.
A. Attitude equations for an inertially pointing satellite with reaction wheels and magnetorquers

The total angular momentum of the satellite in the bodyfixed frame comprises the angular momentum $J \omega$ of the central body and the angular momentum $h_{w}$ of the reaction wheels:

$$
h_{T}=J \omega+h_{w},
$$

where $\omega \in \mathbb{R}^{3}$ and $J \in \mathbb{R}^{3 \times 3}$ are the rotational speed of the satellite body-fixed frame with respect to the inertial frame and the symmetric matrix corresponding to its moment of inertia, respectively. Applying Newton's theorem in the inertial frame, we get

$$
\dot{h}_{T}^{[I]}=T_{m}^{[I]},
$$

where $T_{m}^{[I]}$ is the torque generated by the magnetorquers and acting as an external torque on the spacecraft. When expressed in the body-fixed frame, (2) becomes:

$$
\dot{h}_{T}+\omega^{\times} h_{T}=T_{m},
$$

where the gyroscopic term $\omega^{\times} h_{T}$ appears due the rotation of the body-fixed frame with respect to the inertial frame. Replacing $h_{T}$ in (3) by its expression given in (1) and recognizing that the variation of $h_{w}$ corresponds to the torque $\tau_{w}$ applied by the reaction wheels, with straightforward manipulations, we get the dynamic equations in the body-fixed frame:

$$
\left\{\begin{array}{l}
J \dot{\omega}+\omega^{\times}\left(J \omega+h_{w}\right)=-\tau_{w}+T_{m} \\
\dot{h}_{w}=\tau_{w} .
\end{array}\right.
$$

The attitude of the spacecraft is conveniently described using quaternion coordinates $q=\left[\begin{array}{l}\varepsilon \\ \eta\end{array}\right] \in \mathbb{S}^{3}$, where $\varepsilon \in \mathbb{R}^{3}$ and $\eta \in \mathbb{R}$. By definition, the three-sphere $\mathbb{S}^{3}$ refers to the set of every element $q \in \mathbb{R}^{4}$ satisfying the constraint $|q|=1$. For an inertially pointing satellite, the quaternion $q$ characterizes the instantaneous rotation of the body-fixed frame with respect to the inertial frame. The angular position dynamics is then described by the following well known kinematic differential equation (see, e.g., [19]):

$$
\dot{q}=F(\omega) q=\frac{1}{2}\left[\begin{array}{cc}
-\omega^{\times} & \omega \\
-\omega^{T} & 0
\end{array}\right] q=\frac{1}{2}\left[\begin{array}{c}
-\omega^{\times} \varepsilon+\eta \omega \\
-\omega^{T} \varepsilon
\end{array}\right],
$$

where $F(\omega)$ is clearly a skew-symmetric matrix.

The magnetic torque $T_{m}$ produced by the magnetorquers originates from the interaction between the local geomagnetic field $\tilde{b}$ and the magnetic momentum ${ }^{1} \tau_{m}$ according to the following relationship $T_{m}=-\tilde{b}^{\times} \tau_{m}$. Then, the magnetic field $\tilde{b}$ is a function of both the location of the spacecraft along its orbit and its attitude. These two dependencies can be separated out writing $\tilde{b}$ as $R(q) \tilde{b}_{\circ}$ where $R(q)$ is the rotation matrix expressing the body-fixed frame with respect to the inertial frame, whose expression clearly depends on the quaternion $q$, and $\tilde{b}_{\circ}$ is the geomagnetic field expressed in the inertial frame:

$$
T_{m}=-\left(R(q) \tilde{b}_{\circ}(t)\right)^{\times} \tau_{m} .
$$

\footnotetext{
${ }^{1}$ The dynamics of magnetic coils can be neglected as it reduces to a very fast electrical transient [23].
} 
An explicit expression of $R(q)$ can be written as follows, [16]:

$$
R(q)=\left(\eta^{2}-\varepsilon^{T} \varepsilon\right) I_{3}+2 \varepsilon \varepsilon^{T}-2 \eta \varepsilon^{\times} .
$$

Under the assumption that the orbital trajectory is perfectly known, the time evolution of $\tilde{b}_{\circ}(t)$ can be predicted using a model of the geomagnetic field such as the International Geomagnetic Reference Field (IGRF) which gives access to the local magnetic field at any point in space [39].

\section{B. Global asymptotic stabilization of the attitude dynamics}

In this paper, we will address control design problems for model (4) derived in the previous section aiming at global asymptotic stabilization of the attitude dynamics. This problem can be understood as the problem of designing a feedback controller from $(\omega, q)$ capable of globally ${ }^{2}$ asymptotically stabilizing a suitable equilibrium for the simpler model given by (4b) and

$$
J \dot{\omega}+\omega^{\times} J \omega=u+d
$$

from the control input $u$. The disturbance input $d$ will be useful later and should be considered as zero for stability purposes.

Despite its apparent simplicity, this problem involves tricky aspects that have been highlighted, e.g., in [1]. The main conclusion is that there exists no continuous time-invariant (static or dynamic) controller that globally asymptotically stabilizes a desired equilibrium attitude. This property is related to the fact that every continuous time-invariant closed-loop vector field on $\mathrm{SO}(3)$, the set of attitudes of a rigid body, has more than one closed-loop equilibrium, and hence, the desired equilibrium cannot be globally attractive [1].

However, as soon as the control law is not continuous or time-invariant, this obstruction does not hold anymore and many remarkable solutions to the global attitude stabilization problems have been given in the literature (see, e.g. the recent work [30] and references therein). In this paper, we will use a dynamic (discontinuous) hybrid controller that was recently proposed in [26] whose dynamical equation can be written as follows using the notation in [13], [14]:

$$
\begin{aligned}
& \dot{x}_{c}=0, \quad\left(q, \omega, x_{c}\right) \in C \\
& x_{c}^{+}=-x_{c}, \quad\left(q, \omega, x_{c}\right) \in D \\
& u=-c x_{c} \varepsilon-K_{\omega} \omega
\end{aligned}
$$

where the flow set $C$ and the jump set $D$ are defined as

$$
\begin{aligned}
& C:=\left\{\left(q, \omega, x_{c}\right) \in \mathbb{S}^{3} \times \mathbb{R}^{3} \times\{-1,1\}: x_{c} \eta \geq-\delta\right\} \\
& D:=\left\{\left(q, \omega, x_{c}\right) \in \mathbb{S}^{3} \times \mathbb{R}^{3} \times\{-1,1\}: x_{c} \eta \leq-\delta\right\} .
\end{aligned}
$$

Note that the scalar controller state $x_{c}$ is constrained to belong to the set $\{-1,1\}$ by the hybrid dynamics. Moreover, the control input $u$ corresponds to a state feedback from $(\omega, \varepsilon)$ where the sign of the $\varepsilon$ gain is toggled by $x_{c}$. The suggestive

\footnotetext{
${ }^{2}$ Note that we actually consider a strict subset of $\mathbb{R}^{7}$, since $q^{T} q=1$ holds for all times, and thus we shall call this property "asymptotic stability in the large" rather than "global asymptotic stability" - see [22], however for consistency with existing results, such as [1], [26], we use "global asymptotic stability" throughout this paper.
}

result of [26] is that the hybrid controller (6) is able to asymptotically stabilize the set (attractor) $\mathcal{A} \subset \mathbb{S}^{3} \times \mathbb{R}^{3} \times\{-1,1\}$ defined as:

$$
\mathcal{A}=\left\{q_{\circ}\right\} \times\left\{\left[\begin{array}{l}
0 \\
0 \\
0
\end{array}\right]\right\} \times\{1\} \bigcup\left\{-q_{\circ}\right\} \times\left\{\left[\begin{array}{l}
0 \\
0 \\
0
\end{array}\right]\right\} \times\{-1\}
$$

which is the union of two points, corresponding to $q=q_{\circ}$ and $q=-q_{\circ}$ respectively. Remarkably, due to the well known double coverage nature of quaternion coordinates (see e.g. [5]), these two points correspond to the same element of $\mathrm{SO}(3)$, i.e. the same attitude of the satellite. The following lemma states the global asymptotic stability result of [26] with an additional local exponential stability property which will be useful later in this paper.

Lemma II.1. For any positive definite matrix $K_{\omega}=K_{\omega}^{T} \succ 0$ and scalars $c>0, \delta \in(0,1)$, the closed-loop given by (4b), (5) with $d=0$ and (6) is such that the set $\mathcal{A}$ in (7) is globally asymptotically stable (GAS) and locally exponentially stable (LES).

Proof: The proof of GAS is established in [26, Theorem 4.2]. To show LES, first recall from [26] that all solutions to (4b), (5), (6) (with $d=0$ ) are eventually continuous, indeed as noted in [26, top of page 2525], for a suitable Lyapunov function $V$, one has $V^{+}-V=-4 \delta$ and since $V$ is non-negative, there cannot be any jump in the neighborhood of $\mathcal{A}$ where $V<4 \delta$. Therefore, we study LES by only focusing on the flow dynamics and continuous-time LES bounds. Moreover, since the two points in $\mathcal{A}$ are disjoint, then local analysis amounts to analyzing separately the two points (note that since no jumps occur locally, then $x_{c}$ remains constant along local solutions). We carry out the analysis looking at the left one in (7), namely $x_{\circ}=\left(q_{\circ}, 0,1\right)$. The other one follows the same steps and is omitted.

Consider now that the motion in $\mathbb{S}^{3}$ can be studied in the variables $\varepsilon, \omega$ replacing $\eta$ by its constrained value $\eta=$ $\sqrt{1-\varepsilon^{T} \varepsilon}$ (note also that $\eta$ is positive around the equilibrium $x_{\circ}$ ). Then, we can replace (4b) by $\dot{q}=\frac{1}{2}\left[\begin{array}{c}-\omega^{\times} \varepsilon+\sqrt{1-\varepsilon^{T} \varepsilon \omega} \\ -\omega^{T} \varepsilon\end{array}\right]$ and the linearized dynamics around the equilibrium $x_{\circ}$ corresponds to $\left[\begin{array}{c}\dot{\varepsilon} \\ \dot{\omega}\end{array}\right]=\left[\begin{array}{cc}0 & 1 / 2 \cdot \mathbf{1}_{3} \\ -c J^{-1} & -J^{-1} K_{\omega}\end{array}\right]\left[\begin{array}{l}\varepsilon \\ \omega\end{array}\right]$ which is exponentially stable $^{3}$ for any positive $c$ and positive definite $K_{\omega}$. As a consequence, from the linear approximation theorem (see, e.g., [20, Thm 4.7]), the equilibrium is LES, namely there exist positive scalars $\Upsilon, \lambda$ such that for small enough initial conditions, $|(\varepsilon(t), \omega(t))| \leq \Upsilon \exp (-\lambda t)|(\varepsilon(0), \omega(0))|$ for all $t \geq 0$. Assume now that $\varepsilon(0)$ is small enough so that $\eta(t)=\sqrt{1-\varepsilon(t)^{T} \varepsilon(t)}>0$ for all $t \geq 0$. Then, one can write

$$
\begin{aligned}
|1-\eta| & =(1-\eta) \leq(1+\eta)(1-\eta)=1-\eta(t)^{2} \\
& =\varepsilon(t)^{T} \varepsilon(t) \leq \Upsilon^{2} \exp (-2 \lambda t)|(\varepsilon(0), \omega(0))|^{2}
\end{aligned}
$$

which gives the local exponential bound also for the variable $\eta$ and completes the proof of LES.

\footnotetext{
${ }^{3}$ This fact is easily proven using the Lyapunov function $V=2 c \varepsilon^{T} \varepsilon+$ $\omega^{T} J \omega$ which satisfies $\dot{V}=-2 \omega^{T} K_{\omega} \omega$, and applying La Salle's invariance principle.
} 
Remark 2. One of the reasons why we adopt the hybrid stabilizer of [26] is that the lack of robustness of a nonhybrid stabilizer, together with the so-called unwinding effect (see, [26, §2] for details) is successfully overcome by the hybrid scheme, in light of the results of [14, Ch. 7]. In particular, since all the data of the hybrid solutions proposed here satisfy the basic assumptions of [14] and the attractor (7) is compact, then by applying [14, Lemma 7.20 and Thm 7.21], asymptotic stability is robust to small disturbances and semiglobally practically robust to large ones. In particular, according to the definition in [14, Def. 7.18] all solutions to the dynamical system generated by perturbing the right hand side of the differential equation remain arbitrarily $\delta$-close to the attractor (7) as long as they start in a $\Delta$-ball around it, where as the size of the disturbance shrinks, one can show that $\delta$ becomes arbitrarily small (practical) and $\Delta$ becomes arbitrarily large (semiglobal). For more details, the reader is referred to [14].

Throughout the paper, we will consider the attitude stabilizer (6) for the spacecraft dynamics. However, we stress that the results presented here are valid for any static or dynamic stabilizer inducing GAS and LES of the attractor $\mathcal{A}$ in (7).

\section{Mathematical formalization of the design goal}

While the stabilization result in Lemma II.1 should be regarded as an important step toward the stabilization of dynamics (4), it is still not implementable for that dynamics as the availability of $u$ is an abstraction. Indeed, global asymptotic stabilization of a suitable attractor for (7) requires to reach the attitude equilibrium and to dump the momentum $h_{w}$ of the reaction wheels. If using the magnetorquers for this secondary task, complication arises from the fact that magnetorquers are associated with tricky time-varying controllability problems. Indeed, at any given instant time, the achievable torque $T_{m}$ is constrained to a plane because the vector $T_{m}$ arises from the cross product between $\tau_{m}$ and $\tilde{b}$ and therefore is always orthogonal to $\tilde{b}$. The fact that the $3 \times 3$ matrix $\tilde{b}^{\times}$is structurally singular originates from this remark. For this reason, the preliminary feedback

$$
\tau_{m}=\frac{\tilde{b}^{\times}}{|\tilde{b}|^{2}} u_{m}
$$

is often used and introduces the new control vector $u_{m} \in$ $\mathbb{R}^{3}$ (see e.g. [24] and references therein) so that the resulting torque $T_{m}$ is equal to $-\tilde{b}^{\times} \tilde{b}^{\times} /|\tilde{b}|^{2} u_{m}$. In such a case, the identity $-\tilde{b}^{\times} \tilde{b}^{\times} /|\tilde{b}|^{2}=\mathbf{1}_{3}-\tilde{b} \tilde{b}^{T} /|\tilde{b}|^{2}$ allows to interpret $T_{m}$ as the projection of $u_{m}$ on the orthogonal plane to $\tilde{b}$. Thus, the control action (8) normalizes the magnetic field $\tilde{b}$ and reduces the control effort by canceling out the useless part of $u_{m}$ which is in the direction of $\tilde{b}$.

Despite these limitations, strong controllability properties still exist whenever $\tilde{b}(t)$ is time-varying ${ }^{4}$ and exhibits a sufficiently rich behavior in such a way to being capable

\footnotetext{
${ }^{4}$ Note that it is also possible to achieve 3 -axis stabilization by using only magnetorquers, as demonstrated in [24]. However, only poor attitude control performance can be expected when using this type of solution because of the very nature of these singular actuators.
}

of persistently spanning all of the three-dimensional space. Sufficient properties for the time-varying function $t \mapsto \tilde{b}(t)$ to be able to preserve this controllability can be expressed in terms of the following matrix:

$$
\Pi(t)=\frac{1}{t} \int_{0}^{t} b_{\circ}(\tau) b_{\circ}^{T}(\tau) d \tau,
$$

where $b_{\circ}(t)=\tilde{b}_{\circ}(t) /\left|\tilde{b}_{\circ}(t)\right|$ is the normalized expression of the geomagnetic field in the inertial frame. These properties are formalized in the next assumption and suitably commented next.

Assumption II.1. Given the matrix function $t \mapsto \Pi(t)$ in (9), the following limit exists, is finite and satisfies:

$$
\Pi_{\infty}=\lim _{t \rightarrow+\infty} \Pi(t), \quad \Pi_{\infty} \prec \boldsymbol{1}_{3} .
$$

Moreover, there exists a scalar $\sigma>0$ such that

$$
|M(t)|=\left|t\left(\Pi(t)-\Pi_{\infty}\right)\right| \leq \sigma, \quad \forall t \geq 0 .
$$

It is interesting to remark that if $b_{\circ}(\tau)$ is a signal having finite power then assumption II.1 is verified [42, Chapter 4].

Remark 3. In our preliminary work [37] we imposed a stronger assumption on the magnetic field $b_{\circ}$ because we required it to be periodic with half of the period $T_{0}$ of the orbital motion of the spacecraft. In that specific case, the limit in (10) exists and corresponds to $\Pi_{\infty}=\int_{0}^{T_{0} / 2} b_{\circ}(\tau) b_{\circ}^{T}(\tau) d \tau$ and then boundedness of $M(t)$ can be established by noticing that $\Pi\left(k T_{0} / 2\right)=\Pi_{\infty}$ for each $k \in \mathbb{Z}$ and using boundedness of $b_{\circ}$. Here we impose milder assumptions on $b_{\circ}$ also in light of the data reported later in Figure 5, where the actual evolution of $b_{\circ}$ along several orbits is compared to its periodic approximation.

It is also worthwhile to mention that our Lyapunov construction is inspired by the work of [24], where only the following assumption is made on the geomagnetic field $b_{\circ}$ (see the first displayed equation in [24, Lemma 1]):

$$
\bar{\Gamma}_{0}=\lim _{T \rightarrow+\infty}-\frac{1}{T} \int_{0}^{T} b_{\circ}^{\times}(\tau) b_{\circ}^{\times}(\tau) d \tau, \quad 0<\bar{\Gamma}_{0}<\mathbf{1}_{3} .
$$

Property (12) is weaker than Assumption II.1 and corresponds to only enforcing relation (10), as one can easily check by selecting $\bar{\Gamma}_{0}=\mathbf{1}_{3}-\Pi_{\infty}$. In [24, Proof of Proposition 1] it is claimed that under the assumption that $\left|b_{\circ}(t)\right|=1$ for all $t \geq 0$, (12) is sufficient for the existence of $\sigma>0$ satisfying (11). Nevertheless, selecting $b_{\circ}(t)=\frac{1}{2(t+1)}\left[\begin{array}{c}\sqrt{3 t^{2}+4 t} \sin (t) \\ \sqrt{3 t^{2}+4 t} \cos (t)\end{array}\right]$, it is possible to prove that (12) (equivalently, (10)) holds while there exists no $\sigma>0$ satisfying (11). The proof of this fact is given in Appendix B.Since we follow the construction of [24, Proposition 1] in our Lyapunov derivations, in light of this example, we explicitly enforce (11) in our Assumption II.1. Note that the example above and in Appendix B does not clearly correspond to a plausible physical scenario and arises from a purely mathematical observation. Finally, we should emphasize that the result in [24, Prop. 1] has been proven later in [25] under milder assumptions and with a different proof technique. Here we use the Lyapunov-based proof technique of [24] that can be effectively employed in our context. 
Based on Assumption II.1, we can now mathematically formalize the engineering problem stated in Problem 1 dealing with the implementation of the attitude controller presented in Section II-B to suitably stabilize the spacecraft dynamics presented in Section II.

Problem 2. Consider the spacecraft described by (4) and satisfying Assumption II.1. Design a state-feedback controller acting on the input $\left(\tau_{w}, \tau_{m}\right)$ which ensures global asymptotic stability of the set $\mathcal{A} \times\left\{h_{w} \in \mathbb{R}^{3}: h_{w}=h_{\text {ref }}\right\}$, where $\mathcal{A}$ is defined in (7) and $h_{r e f} \in \mathbb{R}^{3}$ is any constant setpoint reference for the angular momentum $h_{w}$ of the reaction wheels.

\section{A ClASSICAL APPROACH REVISITED}

As a first contribution of this paper, a rigorous mathematical treatment of the so-called "cross product control law" is proposed in Sections III-A and III-B before introducing a revisited version of this control law in Section III-C

\section{A. The classical cross-product control law}

A classical approach to the solution to Problem 2 is the socalled "cross product control law" well surveyed in [3], [4], [8], [33]. This control law has been used, e.g., in the spacecraft Demeter [28], as described in [29]. The underlying philosophy of this approach is to use the reaction wheels actuator for the attitude stabilization loop, while the momentum of the wheels is simultaneously regulated by a second control loop acting on the magnetorquers. This leads to two control loops designed independently, as illustrated next.

1) The attitude stabilization loop: For attitude stabilization, one can rewrite the first relationship in (4a) as

$$
J \dot{\omega}+\omega^{\times} J \omega=\underbrace{-\tau_{w}-\omega^{\times} h_{w}}_{u}+\underbrace{T_{m}}_{d} .
$$

Note that (13) corresponds to (5) with $u=-\tau_{w}-\omega^{\times} h_{w}$ and $d=T_{m}$. Then, forgetting for a moment the presence of the disturbance $d$ (which should however be taken into account at a later stage for stability analysis), the reaction wheels input $\tau_{w}$ can be selected by only focusing on the attitude control goal using some global asymptotic stabilizer, such as the one discussed in Section II-B. In particular, with that construction one can use (6) for $u$ and

$$
\tau_{w}=-\omega^{\times} h_{w}-u .
$$

Then, Lemma II.1 implies that attitude stabilization is achieved globally, with $d=0$.

2) The momentum dumping loop: For the task of controlling $h_{w}$, assume that the attitude controller is capable of converging to (a small enough neighborhood of) the desired equilibrium attitude $(q, \omega) \in \mathcal{A}$ given in (7). The equilibrium, $u+T_{m}=0$ gives $\tau_{w}=T_{m}$, for $(q, \omega) \in \mathcal{A}$. Then, remembering that $h_{r e f}$ is constant, from the second equation in (4a) and from (4c), we get for $(q, \omega) \in \mathcal{A}$,

$$
\widehat{h_{w}} \overline{h_{r e f}}=-\tilde{b}^{\times}(t) \tau_{m} .
$$

One can then select the magnetorquers input $\tau_{m}$ in such a way to stabilize the origin of (15) following, for example, the so-called "cross product control law" of [3], [4], [8], [33], corresponding to

$$
\tau_{m}=-\frac{\tilde{b}^{\times}(t)}{|\tilde{b}(t)|^{2}} k_{p}\left(h_{w}-h_{r e f}\right),
$$

which can be interpreted as the combination of the preliminary feedback (8) with a proportional action $k_{p}\left(h_{w}-h_{r e f}\right)$. It can be shown that this control law globally exponentially stabilizes the attractor

$$
\mathcal{A}_{h}=\left\{h_{w} \in \mathbb{R}^{3}: h_{w}=h_{r e f}\right\}
$$

as stated in the following lemma whose proof, given in Appendix A, follows the same steps as in [24, Prop. 1].

Lemma III.1. If Assumption II.1 holds, then for any scalar $k_{p}>0$, the set $\mathcal{A}_{h}$ in (17) is globally exponentially stable for the closed-loop system (15), (16) with $\tilde{b}$ replaced by $\tilde{b}_{\circ}$.

Remark 4. Following the same philosophy, other control laws have been proposed in the literature to achieve momentum dumping by focusing on (15). For example, in [23], a periodic LQ controller has been designed relying on numerical methods to solve the periodic Riccati equation. Moreover, a semianalytical optimal open-loop solution for only one axis was proposed in [12].

\section{B. Quasi-cascade structure of the classical approach}

A closer look at the "cross product control law" strategy reveals that the attitude stabilization loop (14) is designed assuming that the second loop is at the equilibrium (namely $h_{w}=h_{\text {ref }}$ which induces $d=T_{m}=0$ according to (16) and to (4c)). Conversely, the momentum dumping action (16) is designed assuming that the first loop is at the equilibrium ( $q=q_{\circ}$ o so that $\tilde{b}=R(q) \tilde{b}_{\circ}$ is replaced by $\tilde{b}_{\circ}$ in (15) and (16)). Despite its intuitive interpretation, it seems that formally showing desirable stabilization properties of the overall controller (6), (14), (16) is not so straightforward. Some directions are given with respect to this in [3], [4], [6], [9], [15], [23] where a frequency separation argument is required between the two loops cited above, which corresponds to selecting a very aggressive action of the attitude stabilizer (14). This can be performed, for example, by selecting sufficiently large gains $c$ and $K_{\omega}$ in (6).

One way to tackle the asymptotic stability properties of the equilibrium set $\mathcal{A} \times \mathcal{A}_{h}$ (see (7) and (17)) for the overall dynamics (4), (6), (14), (16) is to recognize that the input $\tau_{w}$ to the reaction wheels does not change the total angular momentum $h_{T}^{[I]}=R^{T}(q)\left(J \omega+h_{w}\right)$ (this follows easily from (1) and (2) and is obvious from the fundamental mechanics viewpoint). Thus, in order to help separating the influence of each actuator, it makes sense to use $h_{T}^{[I]}$, rather than $h_{w}$ to represent the momenta of the reaction wheels in the first loop. Indeed, in the set $\mathcal{A} \times \mathcal{A}_{h}$, one has $h_{T}^{[I]}=R^{T}\left( \pm q_{\circ}\right) h_{w}=h_{w}$. However, when substituting $h_{w}$ by $R(q) h_{T}^{[I]}-J \omega$ in the "cross product control law" (16), bearing in mind that $k_{p}$ is a scalar, 
$(R v)^{\times}=R v^{\times} R^{T}$ for all $v \in \mathbb{R}^{3}$ and $|\tilde{b}|=\left|\tilde{b}_{\circ}\right|$, one gets

$$
\begin{aligned}
\tau_{m}= & -\frac{\left(R(q) \tilde{b}_{\circ}(t)\right)^{\times}}{|\tilde{b}(t)|^{2}} k_{p}\left(R(q) h_{T}^{[I]}-J \omega-h_{r e f}\right) \\
= & -R(q) \frac{\tilde{b}_{\circ}^{\times}(t)}{\left|\tilde{b}_{\circ}(t)\right|^{2}} R^{T}(q) k_{p} R(q)\left(h_{T}^{[I]}\right. \\
& \left.-R^{T}(q)\left(J \omega+h_{r e f}\right)\right) \\
= & -R(q) \frac{\tilde{b}_{\circ}^{\times}(t)}{\left|\tilde{b}_{\circ}(t)\right|^{2}} k_{p}\left(h_{T}^{[I]}-h_{r e f}\right. \\
& \left.+R^{T}(q)(\underbrace{\left(R(q)-\mathbf{1}_{3}\right) h_{r e f}-J \omega}_{\zeta(q, \omega)})\right) .
\end{aligned}
$$

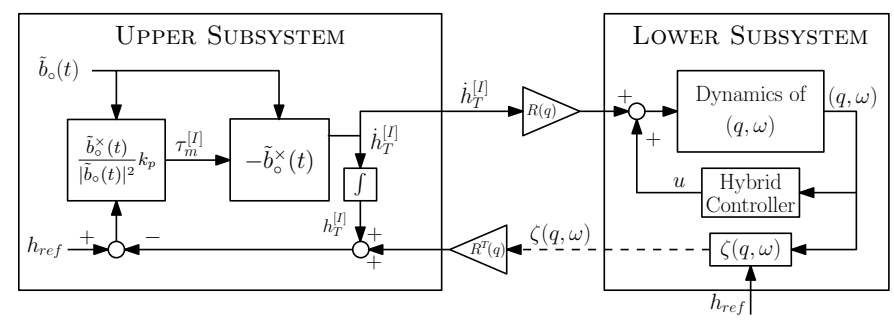

Fig. 2. The quasi-cascaded structure of the "cross product control law" and the role of the signal $\zeta(q, \omega)$.

Equation (18) provides insightful understanding about the quasi-cascaded, or feedback structure of the "cross product control law" which, based on (18) can be remapped in the inertial coordinate frame to get

$$
\tau_{m}^{[I]}=-\frac{\tilde{b}_{\circ}^{\times}(t)}{\left|\tilde{b}_{\circ}(t)\right|^{2}} k_{p}\left(h_{T}^{[I]}-h_{r e f}+R^{T}(q) \zeta(q, \omega)\right) .
$$

This is clarified in the block diagram of Figure 2 where the presence of the signal $\zeta(q, \omega)$ creates an undesirable dependence of $\dot{h}_{T}^{[I]}$ on the attitude parameters $(q, \omega)$. Even though the literature contains reference to frequency separation (or two time scale results when speaking in nonlinear terms), it is unclear how to apply those results to the scheme of Figure 2, where the time-varying input $\tilde{b}_{\circ}(t)$ makes it hard to say that the upper subsystem is slower than the lower one. Conversely, a possible way to establish asymptotic stability of the attractor is via the input-to-state (ISS) small gain approach [17] where one could claim that increasing the gain of the attitude stabilizer in the lower subsystem allows to reduce enough the ISS gain from $h_{T}^{[I]}$ to $R^{T}(q) \zeta$ in the block diagram. Then, one could characterize the "cross product control law" solution as some sort of high-gain feedback solution leading to global asymptotic stabilization of the set $\mathcal{A} \times \mathcal{A}_{h}$ defined in (7) and (17) via small gain results.

\section{A revisited cross-product control law}

While the "cross product control law" approach of the previous section has been long used in experimentation and performs desirably when a high-gain attitude stabilization is adopted, the natural question that arises in light of (18) is whether the signal $\zeta(q, \omega)$ is really necessary. The answer is no, if one recalls that the stabilizer (16) was designed by focusing on the case $(q, \omega) \in \mathcal{A}$ and that in this set one has $\zeta(q, \omega)=0$. Consequently, this signal $\zeta$ can be freely inserted into (16) to obtain the "revisited cross-product control law":

$$
\tau_{m}=-\frac{\tilde{b}^{\times}(t)}{|\tilde{b}(t)|^{2}} k_{p}\left(h_{w}-h_{r e f}-\zeta(q, \omega)\right),
$$

which can be used as an alternative to stabilizer (16). The advantage of using the revisited law (20a) in place of the classical one (16) is best appreciated by rewriting (20a) as follows, by using the identities in (18):

$$
\tau_{m}^{[I]}=-\frac{\tilde{b}_{\circ}^{\times}(t)}{\left|\tilde{b}_{\circ}(t)\right|^{2}} k_{p}\left(h_{T}^{[I]}-h_{r e f}\right) .
$$
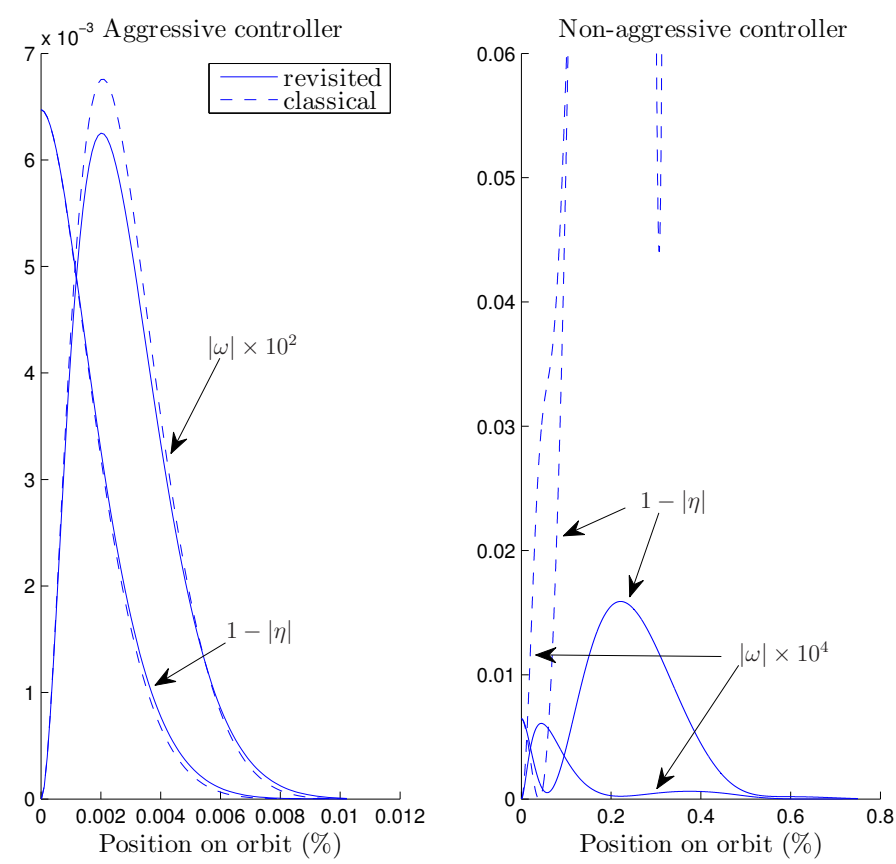

Fig. 3. Comparison in Remark 5 between the classical (dashed) and revisited (solid) "cross-produce control laws" when using an aggressive attitude controllers (left-hand side plot) and a non-aggressive one (right-hand side plot).

Remark 5. From an engineering viewpoint, the revisited control strategy given by (6), (14) and (20) is not much different from the classical "cross-product control law" (6), (14), (16) whenever the attitude stabilizer is aggressive enough so that the standing assumptions behind this historical approach are respected. However, significant differences are witnessed when using non aggressive actions in the attitude controller and this mainly because while the revisited solution keeps performing desirably, the classical solution exhibits very large overshoots and even diverging responses. This can be motivated by the fact that the interaction between the momentum dumper (upper subsystem) and the attitude stabilizer (lower subsystem) in Figure 2 does not anymore satisfy a small gain condition and asymptotic stability is lost.

Figure 3 illustrates this fact by comparatively showing the responses of the two closed-loops starting from the initial conditions $w_{0}=\mathbf{0}, \quad q_{0}=[0.10,0.05,0.02,0.99]^{T}, h_{w 0}=$ $[0.09,0.072,0.078]^{T}, x_{c_{0}}=1$. For illustration purposes, the simulations of Figure 3 have been carried out without imposing 
any saturation limits on $\tau_{w}$ and $\tau_{m}$, so that the difference among the two dynamic solutions can be best appreciated. Simulations incorporating these saturations are carried out in Section V. In the figure, the dashed lines correspond to the response obtained from the classical solution (6), (14), (16) and the solid lines correspond to the revisited law (6), (14) and (20). With these two controllers, two cases are analyzed: the left-hand side plot shows the responses obtained when using an aggressive attitude controller, corresponding to $K_{w}=5 \cdot \mathbf{1}_{3}$ and $c=0.5$ while the right plot corresponds to the less aggressive gains $K_{w}=0.07 \cdot \mathbf{1}_{3}, c=10^{-4}$. In both cases, we consider $\delta=0.5$ and $k_{p}=0.01$. We illustrate the closedloop responses by showing the (rescaled) norm of the angular speed and the scalar value $1-|\eta|$ which is zero in the attractor. Similar comparison results are experienced when looking at all the components of the state $\left(q, \omega, x_{c}, h_{w}\right)$. Note that the responses of the two closed-loops are essentially the same in the case when the classical solution performs desirably, but the revisited solution preserves asymptotic convergence to the attractor, with the less aggressive attitude stabilizer.

One advantage arising from replacing (16) by (20) is that the feedback interconnection of Figure 2 becomes a cascaded interconnection (the dashed arrow disappears) and one can then use results on stability of cascaded nonlinear systems to establish global asymptotic stability of the set $\mathcal{A} \times \mathcal{A}_{h}$ defined in (7) and (17). In particular, for the cascaded system of Figure 2, the upper subsystem regulates the total angular momentum using the magnetorquers and is completely independent from the satellite attitude and the lower subsystem regulates the attitude dynamics using the reaction wheels and is disturbed by the upper subsystem through the action of $d=T_{m}=-R(q) \tilde{b}_{\circ}(t)^{\times} \tau_{m}^{[I]}$. More specifically, the overall closed-loop corresponds to the "revisited cross-product control law" (6), (20) together with

$$
\begin{aligned}
& \dot{h}_{T}^{[I]}=-\tilde{b}_{\circ}(t)^{\times} \tau_{m}^{[I]} \\
& \left\{\begin{array}{l}
(4 \mathrm{~b}) \\
J \dot{\omega}+\omega^{\times} J \omega=u+R(q) \dot{h}_{T}^{[I]} .
\end{array}\right.
\end{aligned}
$$

Using the result established by Lemma III.1, which can be rewritten in the same form when looking at the feedback (21a), (20b), one gets global exponential stability of the upper subsystem. Then, Global Asymptotic Stability can be derived from properties of cascaded interconnections of hybrid systems (recall that the attitude controller is hybrid). The result is formalized in the following theorem.

Theorem III.1. Under Assumption II.1, given any set of gains $k_{p}>0, K_{\omega}=K_{\omega}^{T} \succ 0, c>0$ and $\delta \in(0,1)$, consider the closed-loop system between plant (4) and controller (6), (14), (20a) with state space variables $x=\left(q, \omega, x_{c}, h_{w}\right)$. Then, the set $\mathcal{A}_{e}=\mathcal{A} \times \mathcal{A}_{h}$ as defined in (7) and (17) is locally asymptotically stable.

Moreover, if the hybrid closed-loop system (4b), (5), (6) is ISS from the input $d$ (in the sense of [2]), then the set $\mathcal{A}_{e}$ is globally asymptotically stable, namely the control scheme solved the formalization in Problem 2 of the engineering Problem 1.
Proof: First let us represent the closed-loop with the coordinates $\bar{x}=\left(q, \omega, x_{c}, h_{T}^{[I]}\right)$ and note that, from (1) and $h_{T}^{[I]}=R^{T}(q) h_{T}$, we have $x \in \mathcal{A}_{e}$ if and only if $\bar{x} \in \mathcal{A}_{e}$. Then, we can prove the theorem by studying asymptotic stability of $\mathcal{A}_{e}$ for the transformed dynamics (6), (20b) (21) which is in cascaded form.

For this cascade, using Lemma III.1 rewritten by replacing $h_{w}$ by $h_{T}^{[I]}$, the set $\mathcal{A}_{h}$ is globally exponentially stable for the upper subsystem, corresponding to (21a), (20b). Moreover, the lower subsystem with zero input, corresponding to (6), (21b) with $\dot{h}_{T}^{[I]}=0$, coincides with (4b), (5), (6) with $d=\mathbf{0}$ and therefore guarantees global asymptotic stability (and local exponential stability) of the set $\mathcal{A}$ from Lemma II.1. Then, we can apply [13, Corollary 19] and the follow-up results about the cascades [13, eqn. (23)] to prove local asymptotic stability of the cascade ${ }^{5}$. Moreover, if the lower subsystem is inputto-state stable with respect to $d$ in the sense of [2], then all solutions are bounded for any initial condition. Then, the result follows once again from the results on cascaded system stated for [13, eqn. (23)].

\section{USING STATIC ALLOCATION TO INVERT THE CASCADE}

The solution to Problem 2 presented in the previous section can lead to satisfactory closed-loop responses, however it suffers from three main drawbacks.

1) Due to the cascaded structure of Figure 2, the attitude control loop is undesirably disturbed by the momentum dumping system. Since attitude control is more important than momentum dumping, it would be more desirable if the converse relation was in place.

2) Global asymptotic stability is only established by Theorem III. 1 under an ISS assumption on the attitude control system, which is not easy to guarantee in general. It would be more desirable to have a solution to Problem 2 which is effective without requiring anything more than GAS and LES of the attractor for the attitude control system.

3) The solution should be implemented using (20a) that, through $\zeta(q, \omega)$, requires exact knowledge of the inertia $J$ and may result in lack of robustness if $J$ is uncertain.

The limitations above can be overcome by following an alternative paradigm for the design of the stabilizing law, which arises from the intuition of reversing the cascaded structure of Figure 2. This inversion of the cascade would result in the fact that the attitude stabilization would not be disturbed at all by the momentum dumping. In order to give priority to the attitude control goal, we revisit equation (13) and perform a different partition of the terms at the right hand side in such a way that the disturbance $d$ of the equivalent formulation (5) is zero:

$$
J \dot{\omega}+\omega^{\times} J \omega=\underbrace{-\tau_{w}-\omega^{\times} h_{w}+T_{m}}_{u} .
$$

${ }^{5}$ Note that in [26] forward completeness of all maximal solution is proven so all pre-asymptotic stability results are equivalent to asymptotic stability results. 
Then, the attitude dynamics (4b), (22) corresponds to (4b), (5) with $u=-\tau_{w}-\omega^{\times} h_{w}+T_{m}$ and $d=0$. Following the same steps as in the previous section, the reaction wheels input $\tau_{w}$ can then be selected by only focusing on the attitude control goal using some global asymptotic stabilizer, such as the one discussed in Section II-B. In particular, with that construction, one can use (6) and select

$$
\begin{aligned}
\tau_{w} & =-\omega^{\times} h_{w}+T_{m}-u \\
& =-\omega^{\times} h_{w}-\left(R(q) \tilde{b}_{\circ}(t)\right)^{\times} \tau_{m}-u
\end{aligned}
$$

(where we used (22) and (4c)). Then Lemma II.1 implies that attitude stabilization is achieved globally, regardless of the torque $T_{m}$ generated by the magentorquers.

As a next step, we can now focus on the momenta of the reaction wheels, which are indeed affected by the magnetorquers indirectly from the input selection (23). In particular, using the second equation in (4a), selection (23) and the fact that $h_{r e f}$ is constant, we get

$$
h_{w-h_{r e f}}=-\omega^{\times} h_{w}-u-\left(R(q) \tilde{b}_{\circ}(t)\right)^{\times} \tau_{m},
$$

or

$$
\dot{h}_{w}^{[I]}=-R^{T}(q) u-\tilde{b}_{\circ}^{\times}(t) \tau_{m}^{[I]},
$$

which reveals that the momentum of the reaction wheels is indeed affected by the attitude variables due to the presence of $u$ at the right hand side. Remarking that if $(q, \omega) \in \mathcal{A}$ then (24a) reduces to (15), it appears natural to select the magnetorquers input $\tau_{m}$ in such a way to exploit the useful stabilization result of Lemma III.1, namely

$$
\tau_{m}=-\frac{\left(R(q) \tilde{b}_{\circ}(t)\right)^{\times}}{\left|\tilde{b}_{\circ}(t)\right|^{2}} k_{p}\left(h_{w}-h_{r e f}\right),
$$

or

$$
\tau_{m}^{[I]}=-\frac{\tilde{b}_{\circ}^{\times}(t)}{\left|\tilde{b}_{\circ}(t)\right|^{2}} k_{p}\left(h_{w}^{[I]}-R^{T}(q) h_{r e f}\right),
$$

where $k_{p}$ is a positive scalar gain.

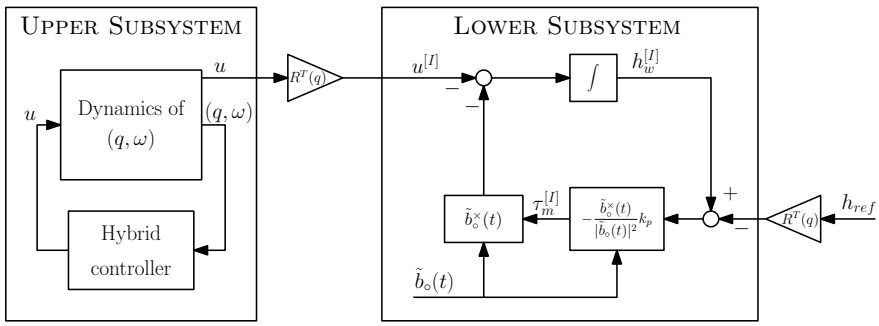

Fig. 4. Cascaded structure of the static allocation scheme.

The structure of the control loop is illustrated in Figure 4 in order to make a thorough comparison with the structure represented in Figure 2. The corresponding equations of the closed-loop system are composed of the control laws (6), (25b) and the upper subsystem

$$
\left\{\begin{array}{l}
(4 \mathrm{~b}) \\
J \dot{\omega}+\omega^{\times} J \omega=u,
\end{array}\right.
$$

which affects the lower subsystem (24b) (25b). This cascaded structure should be compared to (6), (20) and (21). In particular, comparing Figure 4 to Figure 2, it is obvious that we have exchanged the order of the attitude and momentum dumping subcomponents. As mentioned above, an advantage arising from the new cascaded structure of Figure 4 is that the attitude control goal becomes the primary goal and is associated with transient responses that are not disturbed by the momentum dumping controller. Therefore, even though the limit set of the closed-loop trajectories is the same under both controllers, the solution of this section has no impact at all on the transient of the attitude dynamics. This feature is well illustrated by the simulation examples of Section V.

Theorem IV.1. Under Assumption II.1, given any set of gains $k_{p}>0, K_{\omega}=K_{\omega}^{T} \succ 0, c>0$ and $\delta \in(0,1)$, consider the closed-loop system between plant (4) and controller (6), (23), (25a) with state space variables $x=\left(q, \omega, x_{c}, h_{w}\right)$. Then the set $\mathcal{A}_{e}=\mathcal{A} \times \mathcal{A}_{h}$ as defined in (7) and (17) is globally asymptotically stable, namely the control scheme solves the formalization in Problem 2 of the engineering Problem 1.

Proof: The proof uses the results for asymptotic stability of nonlinear hybrid cascaded interconnections that have been recalled in the proof of Theorem III.1 (namely [13, Corollary 19] and the follow-up results about the cascades [13, eqn. (23)]). In particular, generalizing to the hybrid case the classical continuous-time result of [34], global asymptotic stability of the cascaded interconnection can be established by using GAS and LES of the upper subsystem (which holds due to Lemma II.1), 0-GAS of the lower subsystem (namely GAS with zero input) and global boundedness (UGB) of trajectories. These three items are proven below.

O-GES of the lower subsystem. As already said, the zero input equation of the lower subsystem corresponds to (15), (25a) (where it is recalled that $\tilde{b}(t)=R(q) \tilde{b}_{\circ}(t)$ ). Thus, Lemma III.1 can be directly applied to prove that $\mathcal{A}_{h}$ is globally exponentially stable for the lower subsystem with zero input.

Global Boundedness. By global boundedness (GB) of the closed-loop (4), (6), (23), (25a), we mean that for each $r>0$, there exists $\Psi(r)>0$ such that ${ }^{6}$ for each initial condition $x_{0}=\left(\omega_{0}, q_{0}, x_{c 0}, h_{w 0}\right)$ satisfying $\left|x_{0}\right|_{\mathcal{A} \times \mathcal{A}_{h}} \leq r$, one has that all solutions $x$ satisfy $|x(t, j)|_{\mathcal{A} \times \mathcal{A}_{h}} \leq \Psi(r)$ for all $(t, j) \in \operatorname{dom}(x)$. To show this, first note that GAS of the upper subsystem implies GB of the $\left(\omega, q, x_{c}\right)$ substate. As for the state $h_{w}$, consider the function $H=\left|\tilde{h}_{w}\right|^{2} / 2$ where $\tilde{h}_{w}=h_{w}-h_{\text {ref }}$ and note that $\tilde{h}_{w}$ does not change across jumps, while along flows, from (24a) we get:

$$
\begin{aligned}
\dot{H} & =\tilde{h}_{w}^{T}\left(-\omega^{\times}\left(\tilde{h}_{w}+h_{r e f}\right)-\tilde{b}^{\times}(t) \tau_{m}-u\right) \\
& =-\tilde{h}_{w}^{T}\left(u+\omega^{\times} h_{r e f}\right)+k_{p} \tilde{h}_{w}^{T} \frac{\tilde{b}^{\times}(t) \tilde{b}^{\times}(t)}{|\tilde{b}(t)|^{2}} \tilde{h}_{w},
\end{aligned}
$$

indeed $\tilde{h}_{w}^{T} \omega^{\times} \tilde{h}_{w}=-\tilde{h}_{w}^{T} \tilde{h}_{w}^{\times} \omega=0$. Then defining $\rho(t):=|u|+\left|\omega^{\times} h_{r e f}\right|$ and remarking that $-\tilde{b}^{\times}(t) \tilde{b}^{\times}(t)=$ $\left(\tilde{b}^{\times}(t)\right)^{T} \tilde{b}^{\times}(t) \succeq \mathbf{0}, \forall t, \dot{H}$ can be bounded as follows:

$$
\dot{H} \leq\left|\tilde{h}_{w}\right| \rho(t) \leq\left(1+\left|\tilde{h}_{w}\right|^{2}\right) \rho(t)=(1+2 H) \rho(t) .
$$

\footnotetext{
${ }^{6}$ As customary, given a set $\mathcal{S}$ and a vector $w$, we denote the distance of $w$ from $\mathcal{S}$ as $|w|_{\mathcal{S}}:=\inf _{z \in \mathcal{S}}|w-z|$.
} 
From GAS and LES of the $(\omega, q)$ dynamics, for each $r>0$ there exist positive $K_{r}, \lambda_{r}$ such that $\rho(t) \leq K_{r} \exp \left(-\lambda_{r} t\right)$. Then, from Gronwall-Bellman's inequality [20, Lemma A.1], since $\rho(t)$ is integrable, we have that $H=\left|\tilde{h}_{w}\right|^{2} / 2$ is globally bounded.

Let us now summarize the important advantages of using the new allocation-based scheme of this section as compared to the two approaches presented in Section III:

1) Unmodified transient of the attitude dynamics. Bearing in mind that the scheme works for any attitude stabilizer inducing GAS and LES, the transient response induced by the preferred attitude stabilizer is preserved by the allocation-based scheme because the controlled attitude dynamics is now the upper (undisturbed) block of the cascaded structure. Note that an interesting property induced by this feature is that the attitude stabilization is independent of $\tilde{b}(t)$ (or $\tilde{b}_{\circ}(t)$ ) which leads to more desirable robustness properties of the closed loop due to an intrinsic decoupling property.

2) Only GAS and LES of the attitude stabilizer is required. As formally stated in the previous Theorem IV.1, global asymptotic stability of the attractor $\mathcal{A}_{e}$ holds under the mild requirement that the attitude stabilizer induce GAS and LES. This shows clear advantages as compared to: 1) the classical approach, for which instability can be observed for an non-aggressive attitude stabilizer inducing GAS and LES, and 2) its revisited version, which requires an extra ISS property from the attitude stabilizer, as stated in Theorem III.1. Note also that GAS of the attractor $\mathcal{A}_{e}$ implies uniform global convergence of the speed of the reaction wheels to the desired reference $h_{r e f}$. Finally, it is also worthwhile mentioning that the stability properties guaranteed for the allocationbased scheme hold globally. In other terms, this means that the proposed strategy can be suitably employed when dealing with either large or small depointings. To our best knowledge, nothing can be said about the classical scheme with respect to this.

3) Dealing with saturations. Regarding saturation of input torques and reaction wheels speed, the allocationbased scheme is preferable as compared to the classical one for two reasons. First, a formal proof of stability demonstrates that, with the allocation-based scheme, $h_{w}$ converges to the arbitrary set point $h_{r e f}$, which, when suitably defined, helps keeping $h_{w}$ away from the saturation bounds and from zero velocity which induces undesirable stiction effect. Secondly, global stability with the new scheme is guaranteed regardless of the aggressiveness of the attitude stabilizer. This allows implementing low-gain attitude controllers, less inclined to induce saturation of $h_{w}, \tau_{w}$ and $\tau_{m}$. Simulation results proposed in the next section confirm that this property does not hold for the classical scheme.

4) Robustness with respect to model parameters. In the case where $\tilde{b}(t)$ is measured on board, the new control law is independent of the model parameters. This was not the case in the revisited version of the classical approach, where $J$ is required ${ }^{7}$. This may be easily shown by comparing (25a) with (20a) where, in this last case, the dependence upon the inertia matrix $J$ comes through the term $\zeta(q, \omega)$. This remark is an argument in favor of the robustness of the proposed allocation scheme as explained, e.g., in [38].

\section{Simulation Results}

To suitably illustrate the control schemes proposed in this paper, we provide in this section a range of numerical simulations. All the simulation tests are carried out using the physical parameters parameters of the satellite Demeter [28] designed and produced by CNES, the French space agency. This spacecraft is the first microsatellite in the Myriade series, a new concept of modular satellite weighing less than $200 \mathrm{~kg}$, flying at an altitude of $710 \mathrm{~km}$, and developed to provide an affordable access to space. Launched on June 29, 2004, the Demeter mission has now come to its end and the satellite was terminated on December 9, 2010. Like most low orbit satellites, Demeter is equipped with reaction wheels and magnetorquers. A star tracker is used to measured its attitude. All numerical data and specifications were provided by CNES [28] and correspond to the ones used for the real design of the control system implemented on board during the flight of Demeter. For simplicity of the exposition, the Demeter example is used here for an inertial pointing mission (as graphically shown in Figure 1), while the real mission needed geocentric pointing. The inertia matrix is given by

$$
J=\left[\begin{array}{ccc}
39.30 & -3.65 & -0.37 \\
-3.65 & 27.15 & -1.45 \\
-0.37 & -1.45 & 46.54
\end{array}\right] \quad\left(\mathrm{kg} \cdot \mathrm{m}^{2}\right) .
$$

The nominal angular momentum of each reaction wheel is chosen to be 0.06 N.m.s, so that the constant vector $h_{\text {ref }}$ is given by $0.06 \cdot[1,1,1]^{T}$. The actuators need to comply with the following constraints:

$$
\begin{gathered}
\left|h_{w}^{i}\right| \leq 2 h_{r e f}^{i}=0.12 \text { N.m.s, } \quad\left|\tau_{w}^{i}\right| \leq 0.005 \text { N.m, } \\
\left|\tau_{m}^{i}\right| \leq 12 \text { A.m }{ }^{2}, \forall i=1,2,3 .
\end{gathered}
$$

The circular orbit considered for the satellite is characterized by an altitude of $660 \mathrm{~km}$, an inclination of $98.23^{\circ}$ and a local time of the ascending node of $22 \mathrm{~h} 15$. From these parameters, the geomagnetic field $\tilde{b}_{\circ}(t)$ is evaluated using the International Geomagnetic Reference Field (IGRF) model. Figure 5 shows the chronograph of $\tilde{b}_{\circ}$ over a time range of 60 orbits, together with its periodic approximation. It is emphasized that in our preliminary work [37] we investigated the stability properties of our schemes under a periodicity assumption for $\tilde{b}_{\circ}$. Figure 5 well illustrates the relevance of the extension, carried out in this paper, to non-periodic instances of $\tilde{b}_{\circ}$. In Figure 5 as well as in all the other figures reported in this section we use the following color codes: the $x$ component is shown in blue, the $y$ component is shown in red and the $z$ component is shown in green.

\footnotetext{
${ }^{7}$ It should be however stated that the original classical "cross-product" control law does not require the knowledge of $J$.
} 


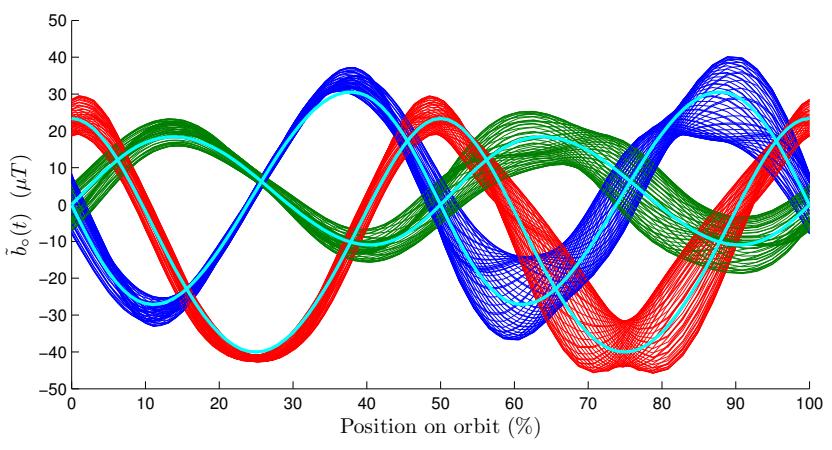

Fig. 5. Chronograph of $\tilde{b}_{\circ}$ together with its periodic approximation (thick cyan line) over 60 orbits.

Several stabilization problems with non-nominal initial angular momentum of the wheels have been simulated using the control laws presented in this paper, namely:

1) the classical approach (dash-dotted lines in our figures);

2) its revisited version (dashed lines);

3) the allocation-based strategy (solid lines).

For all our simulations, unless differently specified, the initial conditions are selected as $h_{w 0}=[0.09,0.05,0.04]^{T}, \omega_{0}=$ 0, $\epsilon_{0}=[0.10,0.05,0.02]^{T}$ and $\eta_{0}=\sqrt{1-\epsilon_{0}^{T} \epsilon_{0}}$.

Regarding the parameter $k_{p}$ inducing momentum dumping, keeping in mind that it should be strictly positive, we ran several numerical simulations of the closed-loop (15) with (16). The resulting choice was $k_{p}=5 \cdot 10^{-3}$. Remarkably, choosing larger or smaller values leads to deteriorated responses, which reveals somewhat interesting trends of the linear time-varying stabilizing law (16).

1) Aggressive attitude stabilizer: As a first test, the parameters of the hybrid attitude stabilizer $u$ defined by (6) are chosen to make this control law aggressive: We assign $K_{\omega}=5 \cdot \mathbf{1}_{3}$ and $c=0.5$. Additionally, $x_{c_{0}}=1$ and $\delta=0.5$. The simulation results are displayed in Figure 6 and Figure 7. While all closed-loop systems eventually converge to the desired attitude equilibrium for all control strategies, it can be seen that all the actuators signals, namely $h_{w}, \tau_{w}$ and $\tau_{m}$, hit the saturation limits (dotted horizontal lines). This is the main drawback of selecting an aggressive attitude stabilizer. Note that using an aggressive attitude controller induces comparable responses on all of the three proposed schemes. We will point out that this is not the case when studying the responses in the presence of disturbances, as illustrated at the end of the section.

2) Non-aggressive attitude stabilizer: In a subsequent simulation test, to prevent saturation, the attitude stabilizer parameters are redefined in order to obtain an attitude controller that is less demanding for the actuators. In particular, we choose the smaller values $c=10^{-4}$ and $K_{\omega}=0.07 \cdot \mathbf{1}_{3}$, while $\delta$ and $x_{c_{0}}$ remain unchanged. The simulation results starting from the same initial conditions are displayed in Figures 8 and 9. From Figure 8, it appears that the classical (dash-dotted) controller leads to instability as one would expect in light of the discussions already provided in Remark 5. Instead, the other control strategies ensure convergence to the equilibrium (as predicted by Theorems IV.1 and III.1) without reaching the actuators limits. Finally, one can observe that the time
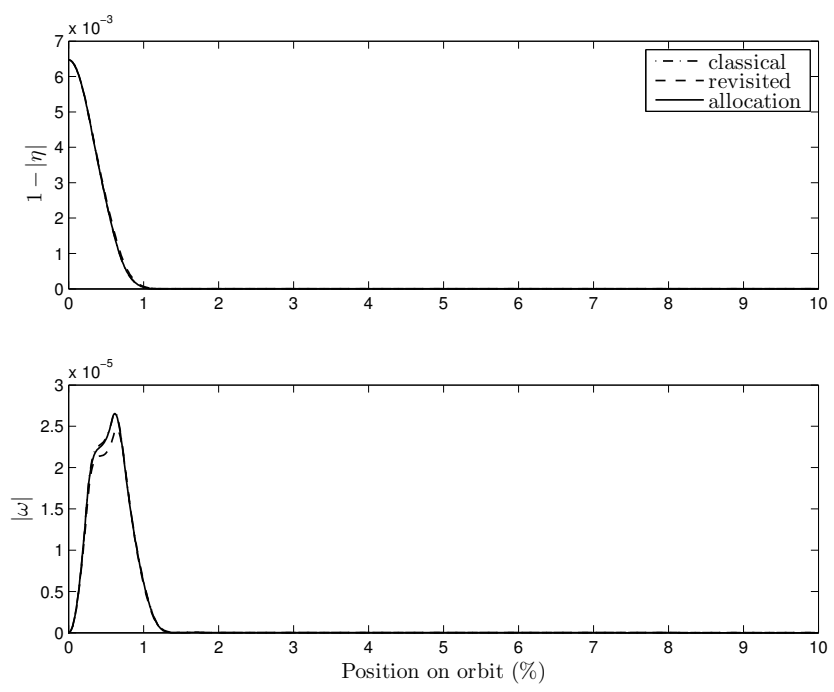

Fig. 6. Quaternion and $\omega$ for the three approaches using an aggressive attitude controller.
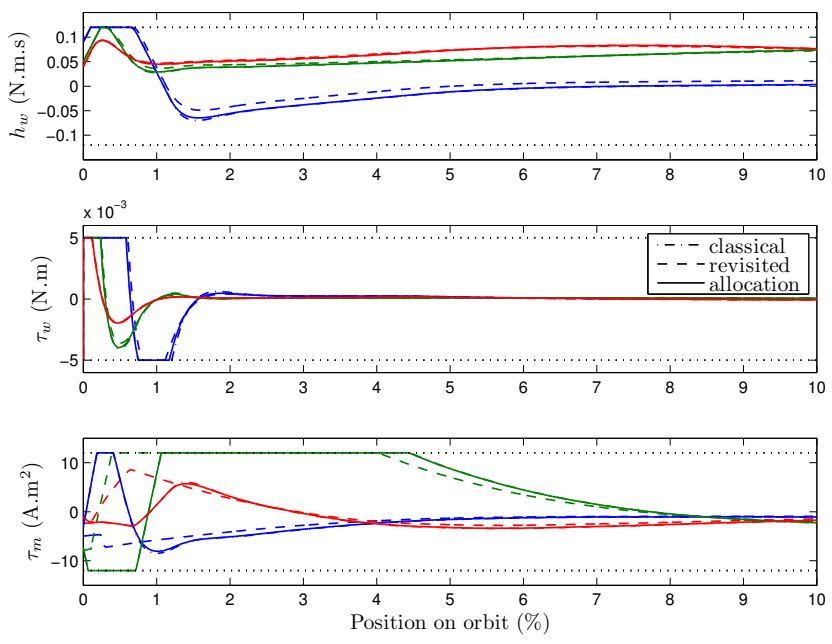

Fig. 7. $h_{w}, \tau_{w}$ and $\tau_{m}$ for the three approaches using an aggressive attitude controller.

evolution of $(\omega, q)$ is more regular with the allocation scheme than it is with the revisited approach. This result is expected as it originates from the hierarchical relationship between the two stabilization tasks, represented in Figure 4, where the attitude control loop is independent of the momentum dumping task so that the allocation control law intrinsically prevents the transient of the reaction wheels desaturation loop from introducing disturbances on the attitude transient.

3) Influence of the uncertainties on the model parameters: Based on the observations already mentioned in Remark 2, the asymptotic stability properties established in this paper are robust to disturbances of the plant dynamics. In light of this fact, using the same non-aggressive attitude stabilizer, a set of Monte-Carlo simulations has been performed to evaluate the sensitivity of each control scheme to variations of $J$. In particular, following the benchmark definition proposed in [28], the Monte-Carlo extractions for the randomized analysis have been carried out using a uniform distribution of the parameters 

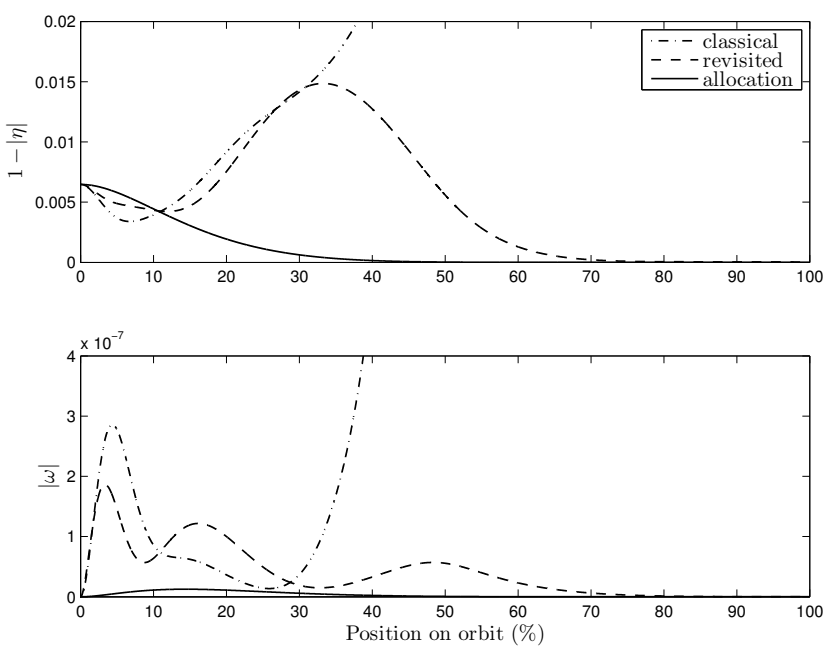

Fig. 8. Quaternion and $\omega$ for the three approaches using a non-aggressive attitude controller.
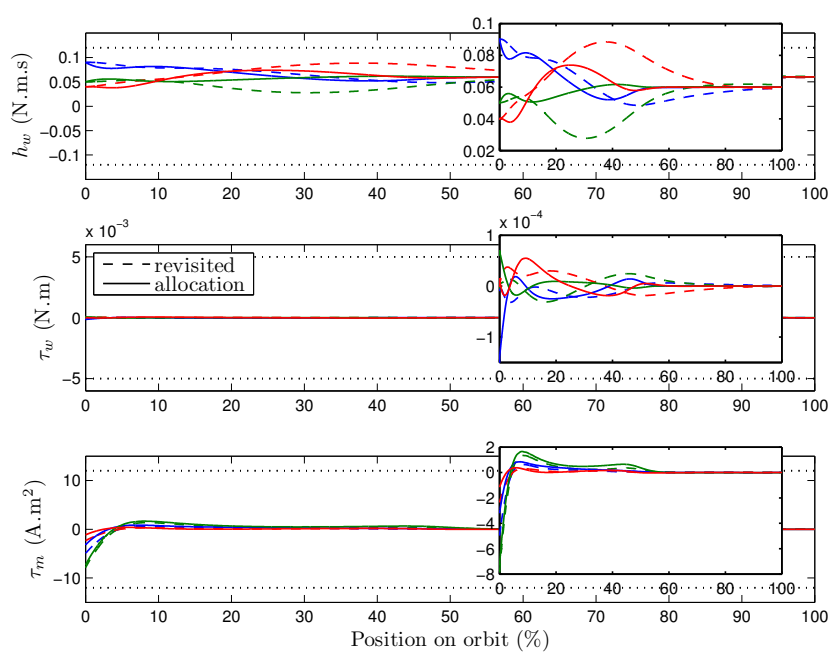

Fig. 9. $h_{w}, \tau_{w}$ and $\tau_{m}$ (with zoom) using a non-aggressive attitude controller for the revisited and the allocation-based strategies.

of $J$ within the following disturbance ranges: $\pm 3 \mathrm{~kg} \cdot \mathrm{m}^{2}$ on the off-diagonal terms and $\pm 30 \%$ on the three diagonal terms. The random disturbed inertia matrix $J$ has been used in place of the nominal one in the satellite dynamics (4) (while the inertia matrix used by the controllers, wherever needed, remains the nominal one). Figures 10 to 13 show the simulation results using 50 such Monte-Carlo extractions for the revisited and the allocation controllers (the response with the classical controller diverges also in this case). Note that larger initial depointings are now considered, namely $\epsilon_{0}=[0.50,0.20,0.05]^{T}$ and $\eta_{0}=\sqrt{1-\epsilon_{0}^{T} \epsilon_{0}}$, while $h_{w 0}=[0.09,0.05,0.04]^{T}$ and $\omega_{0}=\mathbf{0}$ remain the same.

The fact that both of the proposed closed-loop systems behave well and do not reach the saturation limits for all of the considered disturbances of $J$, is a practical illustration of the robustness of these approaches. On the other hand, from a theoretical viewpoint, some level of robustness can be guaranteed from the regularity of the hybrid system data using the results in [14, Chapters 6 and 7] (see also Remark 2). In the case of the allocation-based controller, these observations were predicted by Theorem IV.1 as this control scheme is independent of $J$, which means that closed-loop GAS holds for any positive definite selection of $J$. This is in contrast to the revisited strategy for which the same kind of robustness result seems to be much harder to establish, and might even not hold in general. As for the previous case, one can again observe, by comparing Figures 10 and 12, that the allocation-based solution leads to more regular responses due to the advantages arising from the underlying cascaded scheme that prioritizes the attitude stabilization task.
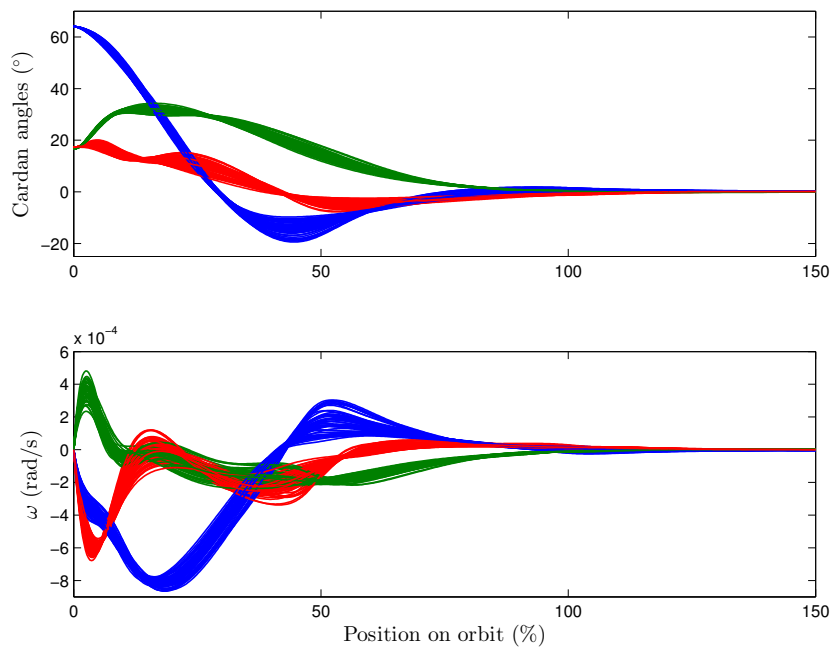

Fig. 10. Monte-Carlo study on uncertainties on $J$ : Cardan angles and $w$ for the revisited controller.
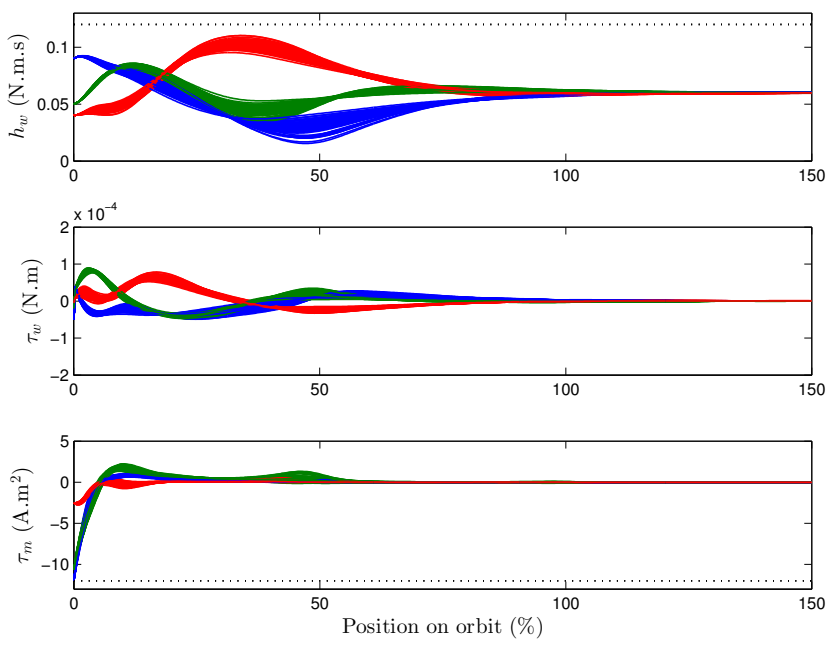

Fig. 11. Monte-Carlo study on uncertainties on $J: h_{w}, \tau_{w}$ and $\tau_{m}$ for the revisited controller.

4) Rejection of periodic disturbances: To assess the performance of the proposed controller in a more realistic environment, a disturbing torque $T_{e x t}$ is injected in the dynamics and comparisons between the classical and the allocation schemes are given via a new set of simulations. Predominant for LEO, as in the Demeter example [28], gravity gradient disturbances, aerodynamic drag and magnetic torques disturbances caused by the interaction of the current loops with the magnetic field, 

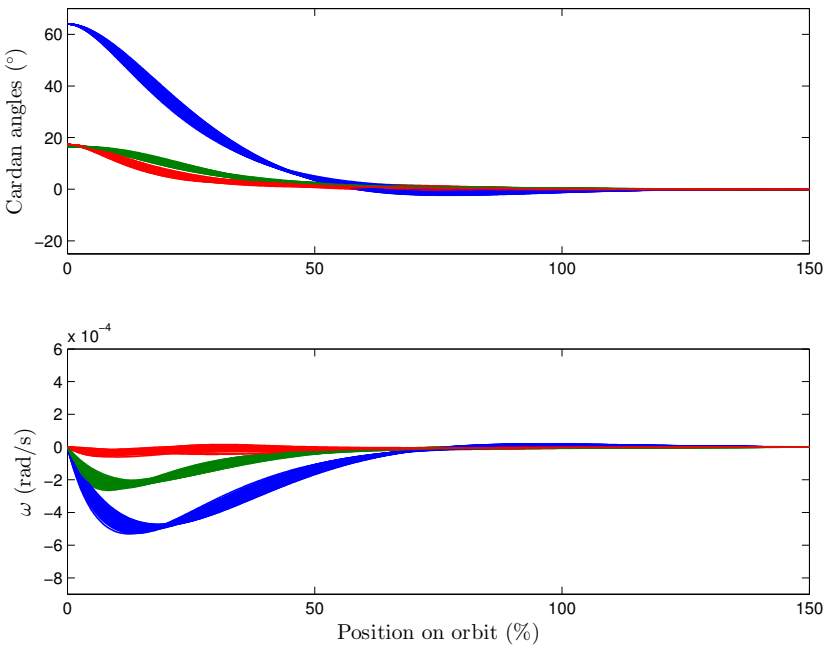

Fig. 12. Monte-Carlo study on uncertainties on $J$ : Cardan angles and $w$ for the allocation-based controller.
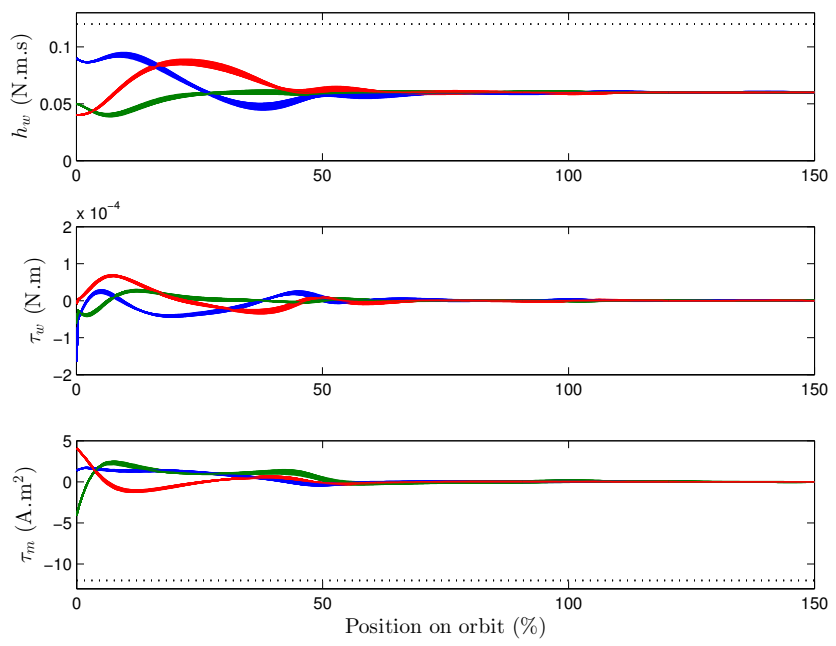

Fig. 13. Monte-Carlo study on uncertainties on $J: h_{w}, \tau_{w}$ and $\tau_{m}$ for the allocation-based controller.

can be modeled in the inertial frame by two periodic signals $T_{1}$ and $T_{2}$, whose period is one and two times the orbital frequency $\omega_{0}$ added to a secular term $T_{0}$ (see [7], [36]):

$$
\begin{aligned}
& T_{e x t}^{[I]}(t)= \\
& {\left[\begin{array}{l}
T_{0 x}+T_{1 x} \sin \left(\omega_{0} t+\varphi_{1 x}\right)+T_{2 x} \sin \left(2 \omega_{0} t+\varphi_{2 x}\right) \\
T_{0 y}+T_{1 y} \sin \left(\omega_{0} t+\varphi_{1 y}\right)+T_{2 y} \sin \left(2 \omega_{0} t+\varphi_{2 y}\right) \\
T_{0 z}+T_{1 z} \sin \left(\omega_{0} t+\varphi_{1 z}\right)+T_{2 z} \sin \left(2 \omega_{0} t+\varphi_{2 z}\right)
\end{array}\right]}
\end{aligned}
$$

The dynamic equation (4a) is then modified accordingly and corresponds to:

$$
J \dot{\omega}+\omega^{\times}\left(J \omega+h_{w}\right)=-\tau_{w}+T_{m}+R(q) T_{e x t}^{[I]}(t) .
$$

Simulations have been performed over 20 orbits using the aggressive attitude stabilizer previously introduced and are reported in Figures 15 and 16. For these simulations, according to [7], [36], the numerical parameteres in (27) have been selected as follows: $T_{0 x}=T_{0 y}=T_{0 z}=1.0 \cdot 10^{-7}$ (N.m), $T_{1 x}=T_{1 y}=T_{1 z}=2.1 \cdot 10^{-5}$ (N.m), $T_{2 x}=$ $T_{2 y}=T_{2 z}=2.1 \cdot 10^{-5}$ (N.m), $\varphi_{T 1 x}=-\varphi_{T 2 x}=\pi / 4$,

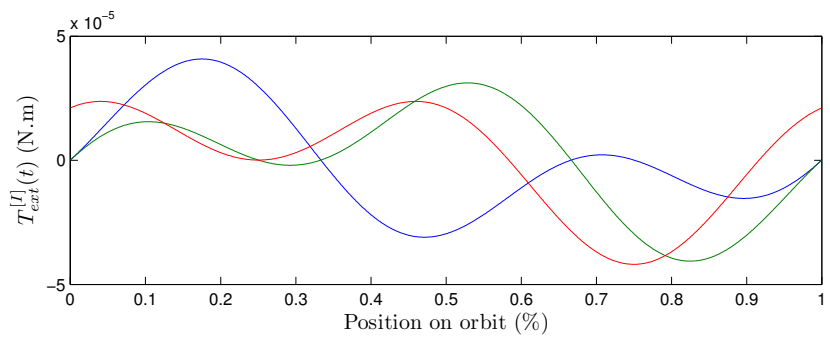

Fig. 14. The periodic disturbance $T_{e x t}^{[I]}$ used for the simulations of Figures 15 and 16 .

$\varphi_{T 1 y}=-\varphi_{T 2 y}=-\pi / 4, \varphi_{T 1 z}=0$ and $\varphi_{T 2 z}=\pi / 2$. This corresponds to the periodic trace for the overall disturbance $T_{e x t}^{[I]}$ represented in Figure 14.

To remain coincise, only the attitude responses variables are displayed in Figures 15 and 16. Indeed, the two control schemes lead to very similar behavior of the actuators signals (namely $h_{w}, \tau_{w}$ and $\tau_{m}$ ) that exhibit an oscillatory behavior that remains well within the saturation limits. The most relevant insight coming from this last set of simulation is revealed by a comparative evaluation of the attitude variables of Figures 15 and 16. Indeed, the advantages arising from the cascaded structure of the allocation-based solution (see Figure 4), where the allocation closed-loop is completely independent of $\tilde{b}_{\circ}$, can be well appreciated in Figure 16, where the attitude is only affected by the periodic external disturbance torque $T_{e x t}^{[I]}$ and the attitude variables converge to a periodic steady-state. Conversely, for the structure in Figure 2 of the classical strategy, the attitude variables are affected by both $T_{e x t}^{[I]}$ and the non-periodic term $\tilde{b}_{\circ}$ (see Figure 5). As a result, the behavior of the attitude variables is non-periodic. Finally, it should be noted that from the two block diagrams of Figures 4 and 2, the attitude of the classical controller is disturbed by its feedback interaction with the momentum dumping dynamics. This fact explains the larger irregular oscillations that can be appreciated by comparing Figures 15 and 16.
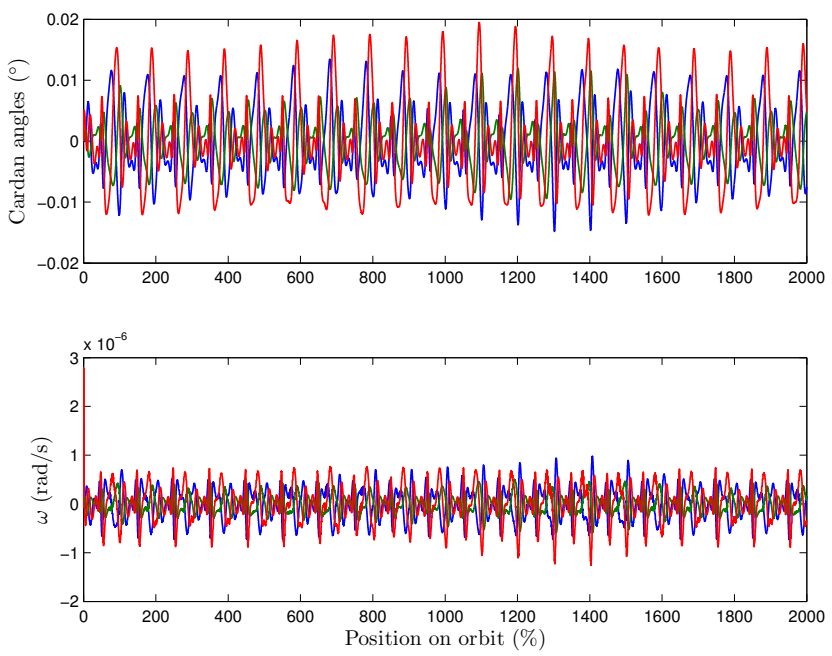

Fig. 15. Attitude reponses (Cardan angles and $w$ ) to a periodic disturbance using the classical controller. 

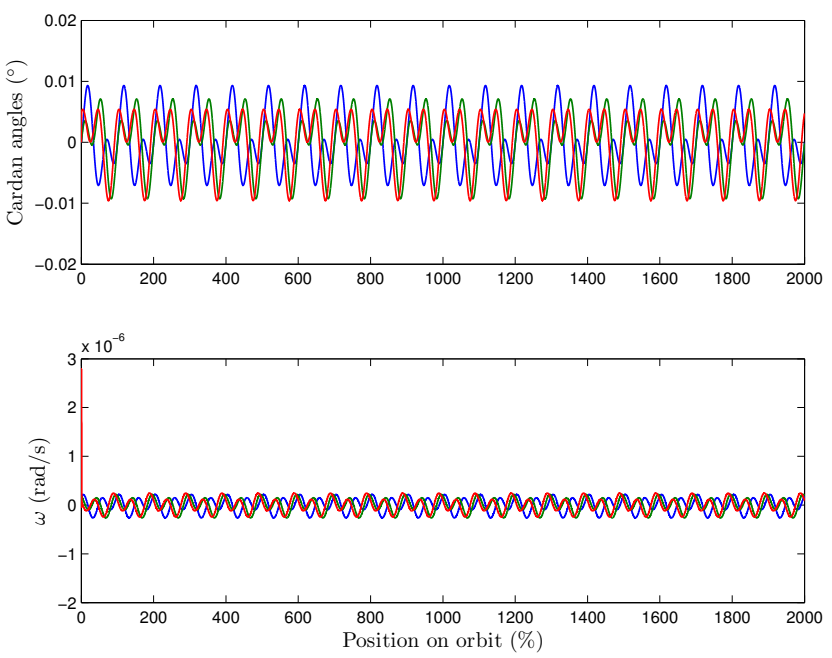

Fig. 16. Attitude reponses (Cardan angles and $w$ ) to a periodic disturbance using the allocation-based controller.

Acknowledgements The authors would like to thank Alessandro Astolfi and Marco Lovera for their insightful comments regarding the observations in Remark 3 and in Appendix B.

\section{Conclusions}

In this paper, the problem of designing an attitude stabilizer for satellites equipped with a set of magnetorquers and reaction wheels is dealt with. The classical "cross product control law" addressing this problem is first investigated and its hidden assumptions are revealed. Then, this control scheme is revisited to obtain a new control strategy benefiting from a rigorous proof of global asymptotic stability and local exponential stability under reasonable assumptions on the geomagnetic field. Relying on this preliminary study, a new allocation-based control scheme is finally proposed as the main contribution of this paper. In addition to the features of the revisited controller, the allocation-based strategy makes the attitude dynamics independent of the momentum dumping task. This is highly desirable as it allows to completely decouple the design of the attitude stabilizer from its implementation problem in satellites with the considered dual actuator configuration.

\section{APPENDIX A}

\section{PROOF OF LEMMA III. 1}

Using $b_{\circ}(t)=\tilde{b}_{\circ}(t) /\left|\tilde{b}_{\circ}(t)\right|$ as defined before Assumption II.1, we can rewrite the closed-loop (15) and (16) with $\tilde{b}$ replaced by $\tilde{b}_{\circ}$ as follows:

$$
\dot{\tilde{h}}_{w}=k_{p} b_{\circ}^{\times}(t) b_{\circ}^{\times}(t) \tilde{h}_{w}
$$

where we use for simplicity $\tilde{h}_{w}=h_{w}-h_{\text {ref }}$. Then in the transformed coordinates, the lemma is proved if we establish UGES of the origin for (29).

We first introduce the candidate Lyapunov function proposed by [24, Prop. 1]

$$
V\left(t, \tilde{h}_{w}\right)=\tilde{h}_{w}^{T}\left(\frac{\lambda}{2} \mathbf{1}_{3}-M(t)\right) \tilde{h}_{w}
$$

where $M(t)$ has been defined in (9)-(11). From the boundedness of $M(t)$, coming from Assumption II.1, and for a large enough $\lambda>0, V$ satisfies, for all $\tilde{h}_{w}$ and for some positive scalars $c_{1}, c_{2}$,

$$
c_{1}\left|\tilde{h}_{w}\right|^{2} \leq V\left(t, \tilde{h}_{w}\right) \leq c_{2}\left|\tilde{h}_{w}\right|^{2}, \quad \forall t \geq 0 .
$$

We can then compute its derivative along dynamics (29) as

$$
\dot{V}\left(t, \tilde{h}_{w}\right)=-\left(\tilde{h}_{w}\right)^{T} Q(t) \tilde{h}_{w}
$$

with

$$
Q(t)=\left(-\lambda \mathbf{1}_{3}+2 M(t)\right) k_{p} b_{\circ}^{\times}(t) b_{\circ}^{\times}(t)+b_{\circ}(t) b_{\circ}^{T}(t)-\Pi_{\infty} .
$$

The two unit vectors $b_{1}(t), b_{2}(t)$ are now introduced. They form an orthogonal basis with $b_{\circ}(t)$, i.e. defining $T(t)=$ $\left[\begin{array}{lll}b_{0}(t) & b_{1}(t) & b_{2}(t)\end{array}\right]$, we have $T^{T}(t) T(t)=\mathbf{1}_{3}$. Then, from the definition of $M(t)$ it is readily seen that $\tilde{Q}(t):=$ $T^{T}(t) Q(t) T(t)$ corresponds to

$$
\begin{aligned}
& \tilde{Q}(t)=\left[\begin{array}{ccc}
1 & 0 & 0 \\
0 & \lambda k_{p} & 0 \\
0 & 0 & \lambda k_{p}
\end{array}\right]-k_{p}\left(\left[\begin{array}{l|l}
0 & \\
0 & \Xi \\
0 &
\end{array}\right]\right. \\
& \left.+\left[\begin{array}{ccc}
0 & 0 & 0 \\
& \Xi^{T}(t)
\end{array}\right]\right)-T^{T}(t) \Pi_{\infty} T(t),
\end{aligned}
$$

with $|\Xi(t)|=\left|T^{T}(t) M(t)\left[\begin{array}{ll}b_{1}(t) \quad b_{2}(t)\end{array}\right]\right| \leq \sigma$. Now, from the rightmost inequality of (10), in Assumption II.1, there exists a positive scalar $\gamma$ such that $\Pi_{\infty} \preceq(1-\gamma) \mathbf{1}_{3}$. Therefore, $T^{T}(t) \Pi_{\infty} T(t) \preceq(1-\gamma) T^{T}(t) T(t)=(1-\gamma) \mathbf{1}_{3}$ and there exists a large enough $\lambda>0$ such that $\tilde{Q}(t) \geq c_{3} \mathbf{1}_{3}$ for some positive scalar $c_{3}$, which implies $Q(t) \geq c_{3} \mathbf{1}_{3}$. Finally, from (32) we get $\dot{V}\left(t, \tilde{h}_{w}\right) \leq-c_{3}\left|\tilde{h}_{w}\right|^{2}$ which, together with (31) implies UGES of the origin for (29) by [20, Thm 4.10].

\section{APPENDIX B}

\section{A PECULIAR SELECTION OF THE GEOMAGNETIC FIELD}

To substantiate the claim given in Remark 3, we prove the next proposition in this appendix, which is aimed at clarifying a subtle issue about the relation between the assumptions in [24, Lemma 1] and our Assumption II.1.

Claim B.1. The following selection:

$$
b_{\circ}(t)=\frac{1}{2(t+1)}\left[\begin{array}{c}
t+2 \\
\sqrt{3 t^{2}+4 t} \sin (t) \\
\sqrt{3 t^{2}+4 t} \cos (t)
\end{array}\right], \quad t \geq 0,
$$

satisfies $\left|b_{\circ}(t)\right|=1$ for all $t \geq 0$, satisfies relation (10) (equivalently (12)) with $\Pi_{\infty}=\frac{1}{8}\left[\begin{array}{lll}2 & 0 & 0 \\ 0 & 3 & 0 \\ 0 & 0 & 3\end{array}\right]$ while there exists no scalar $\sigma>0$ satisfying (11).

To prove Claim B.1 we will make use of the following fact.

Fact B.1. Assume that function $t \mapsto a(t)$ satisfies $\lim _{t \rightarrow+\infty} a(t)=\bar{a}$ for some $\bar{a} \in \mathbb{R}$. Then

$$
L:=\lim _{t \rightarrow+\infty} \frac{1}{t} \int_{0}^{t} a(s) \sin (s) d s=0 .
$$


Proof. Define $\tilde{a}(t)=a(t)-\bar{a}$, so that $\lim _{t \rightarrow+\infty} \tilde{a}(t)=0$. Moreover, given a generic integer $k \geq 0$, define $\tau=t-2 \pi k$ to get

$$
\begin{aligned}
L & =\lim _{t \rightarrow+\infty} \underbrace{\frac{1}{t} \int_{0}^{t} \bar{a} \sin (s) d s}_{\rightarrow 0}+\frac{1}{t} \int_{0}^{t} \tilde{a}(s) \sin (s) d s \\
& =\lim _{t \rightarrow+\infty} \underbrace{\frac{1}{t} \int_{0}^{2 \pi k} \tilde{a}(s) \sin (s) d s}_{\rightarrow 0}+\frac{1}{t} \int_{2 \pi k}^{t} \tilde{a}(s) \sin (s) d s \\
& =\lim _{\tau \rightarrow+\infty} \frac{1}{2 \pi k+\tau} \int_{2 \pi k}^{2 \pi k+\tau} \tilde{a}(s) \sin (s) d s \\
& =\lim _{\tau \rightarrow+\infty} \frac{1}{\tau} \int_{0}^{\tau} \tilde{a}(2 \pi k+\omega) \sin (\omega) d \omega .
\end{aligned}
$$

The proof is completed by using (35) to show that for each positive $\delta>0$, one has:

$$
\limsup _{t \rightarrow+\infty} \frac{1}{t}\left|\int_{0}^{t} a(s) \sin (s) d s\right| \leq \delta,
$$

which clearly implies that the limit in (34) exists and is zero.

To prove (36), since $\tilde{a}$ converges to zero, let us pick $k=k_{\delta}$ sufficiently large to ensure $\left|\tilde{a}\left(2 \pi k_{\delta}+\omega\right)\right|<\delta \forall \omega \geq 0$. Then we have from (35) applied with $k=k_{\delta}$,

$$
\begin{aligned}
& \limsup _{t \rightarrow+\infty} \frac{1}{t}\left|\int_{0}^{t} a(s) \sin (s) d s\right| \\
& =\limsup _{\tau \rightarrow+\infty} \frac{1}{\tau}\left|\int_{0}^{\tau} \tilde{a}\left(2 \pi k_{\delta}+\omega\right) \sin (\omega) d \omega\right| \\
& \leq \limsup _{\tau \rightarrow+\infty} \frac{1}{\tau} \int_{0}^{\tau}\left|\tilde{a}\left(2 \pi k_{\delta}+\omega\right)\right||\sin (\omega)| d \omega \\
& \leq \lim \sup _{\tau \rightarrow+\infty} \frac{1}{\tau} \int_{0}^{\tau} \delta d \omega=\lim \sup _{\tau \rightarrow+\infty} \frac{\delta \tau}{\tau}=\delta,
\end{aligned}
$$

which completes the proof.

In light of Fact B.1 we can now prove Claim B.1.

Proof of Claim B.1. The fact that $\left|b_{\circ}(t)\right|=1, \forall t \geq 0$ can be easily verified by direct calculation.

Moreover, we may easily compute:

$$
\begin{aligned}
& b_{\circ}(t) b_{\circ}^{T}(t)= \\
& {\left[\begin{array}{ccc}
\frac{(t+2)^{2}}{(2 t+2)^{2}} & \star & \star \\
\frac{(t+2) \sqrt{3 t^{2}+4 t}}{(2 t+2)^{2}} \sin (t) & \frac{3 t^{2}+4 t}{(2 t+2)^{2}} \sin ^{2}(t) & \star \\
\frac{(t+2) \sqrt{3 t^{2}+4 t}}{(2 t+2)^{2}} \cos (t) & \frac{3 t^{2}+4 t}{(2 t+2)^{2}} \sin (t) \cos (t) & \frac{3 t^{2}+4 t}{(2 t+2)^{2}} \cos ^{2}(t)
\end{array}\right]}
\end{aligned}
$$

where the $\star$ symbols denote symmetric entries. To compute $\Pi_{\infty}$ we now need to evaluate the asymptotic value of the integral in (10). To this aim, we can apply Fact B.1 to the offdiagonal terms (the sinusoidal terms can be easily transformed in sinuses by a change of variable) to show that they converge to zero. As for the diagonal terms, we can perform a partial fraction expansion of the biproper polynomial factors and use standard formulas to find explicit solutions in terms of suitable combinations sinuses, logarithms and of the "Sine integral" function $S i(t):=\int_{0}^{t} \frac{\sin (s)}{s} d s$, which is known to be globally bounded for all $t$ in $[0,+\infty)$. As a result, we obtain $\Pi_{\infty}=\frac{1}{8}\left[\begin{array}{lll}2 & 0 & 0 \\ 0 & 3 & 0 \\ 0 & 0 & 3\end{array}\right]$, which clearly shows that $\bar{\Gamma}_{0}=\mathbf{1}_{3}-\Pi_{\infty}=$ $\frac{1}{8}\left[\begin{array}{lll}6 & 0 & 0 \\ 0 & 5 & 0 \\ 0 & 0 & 5\end{array}\right]$ satisfies the assumption $0<\bar{\Gamma}_{0}<\mathbf{1}_{3}$ in (12).

Note however that using (11) we have $M(t)=$ $t\left(\Pi(t)-\Pi_{\infty}\right)=\int_{0}^{t} b_{\circ}(\tau) b_{\circ}^{T}(\tau) d \tau-t \Pi_{\infty}=$ $\int_{0}^{t}\left(b_{\circ}(\tau) b_{\circ}^{T}(\tau)-\Pi_{\infty}\right) d \tau$, thus we get that the upper left entry of $M(t)$ corresponds to

$$
\begin{aligned}
M(t)_{[1,1]} & =\int_{0}^{t}\left(\frac{(\tau+2)^{2}}{(2 \tau+2)^{2}}-\frac{1}{4}\right) d \tau=\int_{0}^{t} \frac{2 \tau+3}{4(\tau+1)^{2}} d \tau \\
& =\frac{1}{2}\left(\log (t+1)+\frac{t}{2(t+1)}\right),
\end{aligned}
$$

which clearly grows unbounded as $t$ goes to $+\infty$, thus proving that there exists no scalar $\sigma$ satisfying (11).

\section{REFERENCES}

[1] S.P. Bhat and D.S. Bernstein. A topological obstruction to continuous global stabilization of rotational motion and the unwinding phenomenon. Systems \& Control Letters, 39(1):63-70, 2000.

[2] C. Cai and A.R. Teel. Characterizations of input-to-state stability for hybrid systems. Systems \& Control Letters, 58(1):47-53, 2009.

[3] P.J. Camillo and F.L. Markley. Orbit-averaged behavior of magnetic control laws for momentum unloading. Journal of Guidance and Control, 3(6):563-568, 1980.

[4] C.K. Carrington, W.A. Baracat, and J.L. Junkins. A comparative study of magnetic mometum dump laws. In Proceedings of the AAS/AIAA Astrodynamics Conference, pages 81-139, North Lake Tahoe, Nevada, August 1981.

[5] N.A. Chaturvedi, A.K. Sanyal, and N.H. McClamroch. Rigid-body attitude control. IEEE Control Systems Magazine, 31(3):30-51, 2011.

[6] X. Chen, W.H. Steyn, S. Hodgart, and Y. Hashida. Optimal combined reaction-wheel momentum management for earth-pointing satellites. AIAA Journal of Guidance, Control, and Dynamics, 22(4):543-550, 1999.

[7] M. Courtois. Space Technology Course - spacecraft techniques and technology - Volume 3: Platforms, Module XII: Attitude control and pointing. Cepadues-editions, 2005.

[8] J.H. Decanini, H. Flashner, and H. Schmeichel. Magnetic control and the $25 \mathrm{~kW}$ power system. In AMS Annual Rocky Mountain Guidance and Control Conference, volume 45, page 71, Keystone (CO), USA, 1981.

[9] D. Desiderio, M. Lovera, S. Pautonnier, and R. Drai. Magnetic momentum management for a geostationary satellite platform. IET Control Theory and Applications, 3(10):1370-1382, 2009.

[10] J.R. Forbes and C.J. Damaren. Geometric approach to spacecraft attitude control using magnetic and mechanical actuation. AIAA Journal of Guidance, Control, and Dynamics, 33(2):590-595, 2010.

[11] S. Galeani, A. Serrani, G. Varano, and L. Zaccarian. On linear overactuated regulation using input allocation. In Joint Conference on Decision and Control and European Control Conference, pages 47714776, Orlando (FL), USA, 2011.

[12] F. Giulietti, A. Quarta, and P. Tortora. Optimal control laws for momentum-wheel desaturation using magnetorquers. AIAA Journal of Guidance, Control, and Dynamics, 29(6):1464-1468, 2006.

[13] R. Goebel, R.G. Sanfelice, and A.R. Teel. Hybrid dynamical systems. IEEE Control Systems Magazine, 29(2):28-93, April 2009.

[14] R. Goebel, R.G. Sanfelice, and A.R. Teel. Hybrid Dynamical Systems: modeling, stability, and robustness. Princeton University Press, 2012.

[15] H.B. Hablani. Pole-placement technique for magnetic momentum removal of Earth-pointing spacecraft. AIAA Journal of guidance, control, and dynamics, 20(2):268-275, 1997.

[16] P.C. Hugues. Spacecraft attitude dynamics. Dover Publications, 1986.

[17] Z.P. Jiang, A.R. Teel, and L. Praly. Small-gain theorem for ISS systems and applications. Mathematics of Control, Signals, and Systems (MCSS), 7(2):95-120, 1994.

[18] T.A. Johansen and T.I. Fossen. Control allocation - a survey. Automatica, 49(5):1087-1103, 2013.

[19] S.M. Joshi, A.G. Kelkar, and J.T.Y. Wen. Robust attitude stabilization of spacecraft using nonlinear quaternion feedback. IEEE Transactions on Automatic Control, 40(10):1800-1803, 1995.

[20] H. Khalil. Nonlinear Systems. Prentice Hall, third edition, 2002.

[21] A. Lekka, M.C. Turner, and P.P. Menon. Full and reduced order IMC anti-windup compensators for a class of nonlinear systems with application to wave energy converter control. In Proc. of the American Control Conference, pages 4861-4866. IEEE, 2013. 
[22] A. Loria and E. Panteley. Stability, told by its developers. In A. Loria F. Lamnabhi-Lagarrigue, and E. Panteley, editors, Advanced Topics in Control Systems Theory, volume 328 of Lecture Notes in Control and Information Science, pages 199-258. Springer London, 2006.

[23] M. Lovera. Optimal magnetic momentum control for inertially pointing spacecraft. European Journal of Control, 7(1):30-39, 2001.

[24] M. Lovera and A. Astolfi. Spacecraft attitude control using magnetic actuators. Automatica, 40(8):1405-1414, 2004.

[25] M. Lovera and A. Astolfi. Global magnetic attitude control of inertially pointing spacecraft. AIAA Journal of guidance, control, and dynamics, 28(5):1065-1072, 2005

[26] C.G. Mayhew, R.G. Sanfelice, and A.R. Teel. Robust global asymptotic attitude stabilization of a rigid body by quaternion-based hybrid feedback. In Joint Conference on Decision and Control and Chinese Control Conference, pages 2522-2527, Shanghai, China, 2009.

[27] R.J. McElvain. Satellite angular momentum removal utilizing the Earth's magnetic field. In S.F. Singer, editor, Torques and attitude sensing in Earth satellites, pages 137-158. Academic Press, New York, 1964.

[28] C. Pittet and D. Arzelier. Demeter: a benchmark for robust analysis and control of the attitude of flexible microsatellites. In IFAC Symposium on Robust Control Design (ROCOND), Toulouse, France, 2006.

[29] C. Pittet and C. Fallet. Gyroless attitude control of a flexible microsatellite. In 5th Conference on Dynamics and Control of Systems and Structures in Space (DCSSSO2), Cambridge, UK, 2001.

[30] R. Schlanbusch, A. Loria, and P.J. Nicklasson. On the stability and stabilization of quaternion equilibria of rigid bodies. Automatica, 48(12):3135-3141, 2012.

[31] A. Serrani. Output regulation for over-actuated linear systems via inverse model allocation. In Conference on Decision and Control, pages 48714876, Maui (HI), USA, 2012.

[32] A. Serrani and M. Bolender. Invited session: Control of over-actuated systems: Application to guidance and control of aerospace, marine and terrestrial vehicles. In 14th Mediterranean Conference on Control and Automation, Ancona (Italy), June 2006.

[33] M.J. Sidi. Spacecraft dynamics and control: a practical engineering approach. Cambridge University Press, 1997.

[34] E.D. Sontag. Remarks on stabilization and input-to-state stability. In Conference on Decision and Control, pages 1376-1378, Tampa, Florida, December 1989.

[35] S. Tarbouriech, G. Garcia, J.M. Gomes da Silva Jr., and I. Queinnec. Stability and stabilization of linear systems with saturating actuators. Springer-Verlag London Ltd., 2011.

[36] Jean-François Trégouët, Denis Arzelier, Dimitri Peaucelle, Yoshio Ebihara, Christelle Pittet, and Alexandre Falcoz. Periodic $\mathrm{H}_{2}$ synthesis for spacecraft attitude control with magnetorquers and reaction wheels. In Joint Conference on Decision and Control and European Control Conference, pages 6876-6881, Orlando, FL, USA, 2011.

[37] J.F. Trégouët, D. Arzelier, D. Peaucelle, and L. Zaccarian. Static input allocation for reaction wheels desaturation using magnetorquers. In 19th IFAC Symposium on Automatic Control in Aerospace, Würzburg (Germany), September 2013.

[38] J.T.Y. Wen and K. Kreutz-Delgado. The attitude control problem. IEEE Transactions on Automatic Control, 36(10):1148-1162, 1991.

[39] J.R. Wertz. Spacecraft attitude determination and control. Kluwer Academc Publishers, 1978.

[40] L. Zaccarian. Dynamic allocation for input-redundant control systems. Automatica, 45:1431-1438, 2009.

[41] L. Zaccarian and A.R. Teel. Modern anti-windup synthesis: control augmentation for actuator saturation. Princeton University Press, Princeton (NJ), 2011.

[42] K. Zhou, J.C. Doyle, and K. Glover. Robust and optimal control. Prentice Hall, 1998. 